\title{
Large-amplitude electromagnetic waves in magnetized relativistic plasmas with temperature
}

\author{
V. Muñoz ${ }^{1}$, F. A. Asenjo ${ }^{2}$, M. Domínguez ${ }^{1}$, R. A. López ${ }^{1}$, J. A. Valdivia ${ }^{1}$, A. Viñas ${ }^{3}$, and T. Hada ${ }^{4}$ \\ ${ }^{1}$ Departamento de Física, Facultad de Ciencias, Universidad de Chile, Casilla 653, Santiago, Chile \\ ${ }^{2}$ Departamento de Ciencias, Facultad de Artes Liberales, Facultad de Ingeniería y Ciencias, Universidad Adolfo Ibáñez, \\ Santiago, Chile \\ ${ }^{3}$ NASA Goddard Space Flight Center, Heliophysics Science Division, Geospace Physics Laboratory, Greenbelt, MD, USA \\ ${ }^{4}$ Department of Earth System Science and Technology, Kyushu University, Fukuoka, Fukuoka Prefecture 812-8581, Japan \\ Correspondence to: V. Muñoz (vmunoz@fisica.ciencias.uchile.cl)
}

Received: 16 September 2013 - Revised: 28 November 2013 - Accepted: 6 December 2013 - Published: 14 February 2014

\begin{abstract}
Propagation of large-amplitude waves in plasmas is subject to several sources of nonlinearity due to relativistic effects, either when particle quiver velocities in the wave field are large, or when thermal velocities are large due to relativistic temperatures. Wave propagation in these conditions has been studied for decades, due to its interest in several contexts such as pulsar emission models, laser-plasma interaction, and extragalactic jets.

For large-amplitude circularly polarized waves propagating along a constant magnetic field, an exact solution of the fluid equations can be found for relativistic temperatures. Relativistic thermal effects produce: (a) a decrease in the effective plasma frequency (thus, waves in the electromagnetic branch can propagate for lower frequencies than in the cold case); and (b) a decrease in the upper frequency cutoff for the Alfvén branch (thus, Alfvén waves are confined to a frequency range that is narrower than in the cold case). It is also found that the Alfvén speed decreases with temperature, being zero for infinite temperature.

We have also studied the same system, but based on the relativistic Vlasov equation, to include thermal effects along the direction of propagation. It turns out that kinetic and fluid results are qualitatively consistent, with several quantitative differences. Regarding the electromagnetic branch, the effective plasma frequency is always larger in the kinetic model. Thus, kinetic effects reduce the transparency of the plasma. As to the Alfvén branch, there is a critical, nonzero value of
\end{abstract}

the temperature at which the Alfvén speed is zero. For temperatures above this critical value, the Alfvén branch is suppressed; however, if the background magnetic field increases, then Alfvén waves can propagate for larger temperatures.

There are at least two ways in which the above results can be improved. First, nonlinear decays of the electromagnetic wave have been neglected; second, the kinetic treatment considers thermal effects only along the direction of propagation. We have approached the first subject by studying the parametric decays of the exact wave solution found in the context of fluid theory. The dispersion relation of the decays has been solved, showing several resonant and nonresonant instabilities whose dependence on the wave amplitude and plasma temperature has been studied systematically. Regarding the second subject, we are currently performing numerical 1-D particle in cell simulations, a work that is still in progress, although preliminary results are consistent with the analytical ones.

\section{Introduction}

Due to their interest both in astrophysical and laboratory plasmas, relativistic electron-positron plasmas have been a subject of study for decades (Ruffini et al., 2010). In astrophysics, they are relevant in systems such as accretion disks around black holes (Björnsson et al., 1996; Liang, 1979; 
White and Lightman, 1989), models of the early universe (Gibbons et al., 1985; Tajima and Taniuti, 1990; Tatsuno et al., 2003; Lesch and Pohl, 1992), supernova remnants and active galactic nuclei (Hardy and Thoma, 2000; Reynolds et al., 1996), pulsar magnetospheres (Curtis, 1991; Istomin and Sobyanin, 2007; Manchester and Taylor, 1977; Sturrock, 1971), magnetars (neutron stars with magnetic fields up to $\sim 10^{14} \mathrm{G}$ ) (Beskin et al., 1993), hypothetical quark stars (Usov, 1998), and gamma-ray bursts (Piran, 1999, 2004). Regarding laboratory plasmas, they have been considered in the study of ultra-intense lasers (Blaschke et al., 2006), and in laboratory and tokamak plasmas (Zank and Greaves, 1995). For instance, recent experiments on relativistic electron-positron creation with short ultra-intense laser pulses $\left(\sim 10^{20} \mathrm{~W} \mathrm{~cm}^{-2}\right)$ have been performed (Chen et al., 2009), where measurements indicate the positron density to be $\sim 10^{16} \mathrm{~cm}^{-3}$. Electron-positron pair production is also expected to occur in large tokamaks, where up to about $\sim 10^{14}$ positrons may be created in collisions between runaway electrons and background particles (Helander and Ward, 2003).

Of particular interest are effects related to wave propagation in these plasmas, such as proposed pulsar radio emission processes (Luo et al., 2002), bulk acceleration of relativistic jets (Iwamoto and Takahara, 2002), jet formation (Sawyer, 2008; Wardle et al., 1998), electron-positron pair annihilation into one photon in the presence of a strong magnetic field (Harding, 1986), and also in laboratory environments, in problems such as pair production by optical lasers (Blaschke et al., 2006; Chen et al., 2011).

In several of the environments mentioned above, relativistic effects and temperature play an important role, thus it is fundamental to understand wave propagation modes in relativistic plasmas with temperature. In this paper we will focus on the particular case of circularly polarized electromagnetic waves, which, though simple, allows us to study in detail the effect of relativistic temperatures on wave propagation in relativistic hot plasmas.

First, in Sect. 2 it is shown that an exact solution can be found for the fully relativistic fluid equations, including relativistic temperatures (Asenjo et al., 2009). The dispersion properties of the wave are studied, showing that two branches appear, an Alfvén branch and an electromagnetic one, and also showing that relativistic effects increase the plasma transparency, and confine the Alfvén branch to a lower frequency and wave number range. Then, in Sect. 3 the problem is considered from the point of view of a kinetic model (Domínguez et al., 2012). Although qualitative agreement with the fluid results is found, a major difference between both models is the fact that, in the kinetic one, the Alfvén branch is suppressed if the plasma temperature is large enough.

There are at least two ways in which the above results can be improved. First, nonlinear decays of the electromagnetic wave have been neglected; second, the kinetic treatment con- siders thermal effects only along the direction of propagation. In order to address the first subject, we have also studied the parametric decays of the exact wave solution found in the context of fluid theory (López et al., 2012), results that are shown in Sect. 4. There are several resonant and nonresonant instabilities, whose dependence on the wave amplitude and plasma temperature are studied systematically. Regarding the second subject, thermal effects in all directions with respect to the background magnetic field can be readily taken into account in numerical simulations. Thus, we are currently performing numerical 1-D particle in cell simulations, a work that is still in progress, (López et al., 2014) although preliminary results, shown in Sect. 5, are consistent with the analytical ones. Finally, in Sect. 6 results are summarized and discussed.

\section{Exact solution of relativistic fluid equations}

Exact solutions for the plasma equations can be found for cold nonrelativistic plasmas. For instance, circularly polarized Alfvén electromagnetic waves propagating parallel to an external magnetic field are an exact solution of the magnetohydrodynamic equations even when the amplitude is large (Barnes and Hollweg, 1974). Also, a circularly polarized wave in a multiple ion species plasma with drifts is a finite-amplitude solution of the cold plasma model (Gomberoff et al., 1994). The nonlinear propagation of circularly polarized electromagnetic waves in unmagnetized electron-positron-ion plasmas has also been studied in the cold (Berezhiani and Mahajan, 1994) and relativistically hot (Berezhiani and Mahajan, 1995) cases, showing the existence of stable localized structures.

Here we propose an approach that permits one to find an exact solution for the propagation modes in a relativistic electron-positron plasma with constant, arbitrary temperature, within the context of a fluid theory. This can be done by basing our approach on the magnetofluid field unification formalism (Mahajan, 2003).

In this unification approach, the whole plasma is treated as a unique field where the electromagnetic field is coupled with the charged fluid field through a function that carries statistical information of the system. This leads to a simple and elegant way of describing relativistic plasmas. The formalism has also been used successfully to study wave propagation in relativistic two-fluid plasmas (Soto-Chavez et al., 2010), to find equilibrium states via a variational principle (Pino et al., 2010), and has been extended to non-Abelian fields in order to study quark-gluon plasmas (Bambah et al., 2006).

In this paper, we use the approach presented by Mahajan (2003) to derive the dispersion relation for circularly polarized waves of arbitrary amplitude propagating along a constant magnetic field, for arbitrary temperatures. 


\subsection{Magnetofluid unification}

Usually, the interaction of particles with electromagnetic fields is described by introducing a "minimal coupling" in

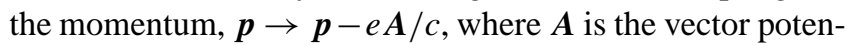
tial. This leads to an energy-momentum conservation equation. This equation and Maxwell equations describe the basic dynamics of charged relativistic particles in plasmas.

It has been suggested (Mahajan, 2003) that the coupling of a relativistic charged fluid, at a given temperature, with the electromagnetic field can be described by an antisymmetric field tensor that contains the statistical information of the system. Thus, the set of equations to describe the plasma dynamics in the homentropic regime, for species $j$, are the Maxwell equations, the continuity equation, and the equation $q_{j} U_{j_{\mu}} M_{j}^{v \mu}=0$.

The field $M_{j}^{\mu \nu}$ is the tensor that couples the electromagnetic and fluid fields. This tensor is defined as $M_{j}^{\mu \nu}=F^{\mu \nu}+$ $\left(m_{j} c^{2} / q_{j}\right) S_{j}^{\mu \nu}$, with $F^{\mu v}$ as the electromagnetic tensor, $q_{j}$ is the charge, and where the new antisymmetric tensor $S_{j}^{\mu \nu}=$ $\partial^{\mu}\left(f_{j} U_{j}^{v}\right)-\partial^{\nu}\left(f_{j} U_{j}^{\mu}\right)$ is introduced, representing the relativistic thermal fluid. Here, $U_{j}^{\mu} \rightarrow\left(\gamma_{j}, \gamma_{j} \boldsymbol{v}_{j} / c\right)$ is the fourvelocity of the fluid, $\boldsymbol{v}_{j}$ is the species velocity,

$\gamma_{j}=\left(1-\frac{\boldsymbol{v}_{j}^{2}}{c^{2}}\right)^{-1 / 2}=\left(1-\frac{\boldsymbol{v}_{j}^{2}}{c^{2}}\right)^{-1 / 2}$,

and $c$ is the speed of light.

The parameter $f_{j}$ is a function of the temperature $T$. An explicit form for $f\left(T_{j}\right)$ can be obtained by assuming a given statistical behavior for the gas. For instance, if the system follows a Maxwell-Jüttner equilibrium distribution (Berezhiani and Mahajan, 1995; Mahajan, 2003),

$f\left(T_{j}\right)=\frac{K_{3}\left(\frac{m_{j} c^{2}}{k_{B} T_{j}}\right)}{K_{2}\left(\frac{m_{j} c^{2}}{k_{B} T_{j}}\right)} \equiv f_{j}$,

where $K_{2}$ and $K_{3}$ are the modified Bessel functions of orders 2 and 3, respectively, and $k_{B}$ is the Boltzmann constant. However, within the treatment carried out in this paper, no explicit description for the function $f$ is needed, and all the analytical and numerical results that follow are independent of the underlying particle distribution function.

Then the plasma dynamics turns out to be given by the momentum equation (Mahajan, 2003)

$$
\left(\frac{\partial}{\partial t}+\boldsymbol{v}_{j} \cdot \nabla\right)\left(f_{j} \gamma_{j} \boldsymbol{v}_{j}\right)=\frac{q_{j}}{m_{j}}\left(\boldsymbol{E}+\frac{1}{c} \boldsymbol{v}_{j} \times \boldsymbol{B}\right)-\frac{1}{m_{j} n_{j}} \nabla p_{j},
$$

which is the spacelike component of Eq. (1), with $p_{j}$ the pressure of species $j$, the pressure equation

$$
\left(\frac{\partial}{\partial t}+\boldsymbol{v}_{j} \cdot \nabla\right) p_{j}=m_{j} \frac{n_{j}}{\gamma_{j}} c^{2}\left(\frac{\partial}{\partial t}+\boldsymbol{v}_{j} \cdot \nabla\right) f_{j},
$$

the continuity equation

$\frac{\partial n_{j}}{\partial t}+\nabla \cdot\left(n_{j} \boldsymbol{v}_{j}\right)=0$,

and Maxwell equations. These yield

$\nabla \cdot \boldsymbol{E}=4 \pi e\left(n_{\mathrm{p}}-n_{\mathrm{e}}\right)$,

$\boldsymbol{\nabla} \times \boldsymbol{B}=\frac{4 \pi}{c} \boldsymbol{J}+\frac{1}{c} \frac{\partial \boldsymbol{E}}{\partial t}$,

and, in the Lorentz gauge,

$\frac{1}{c^{2}} \frac{\partial^{2} \boldsymbol{A}}{\partial t^{2}}-\nabla^{2} \boldsymbol{A}=\frac{4 \pi}{c} \boldsymbol{J}=\frac{4 \pi}{c} \sum_{j} q_{j} n_{j} \boldsymbol{v}_{j}$.

Also, we will consider $p_{j}=n_{j}^{\mathrm{R}} T_{j}$, where $n_{j}^{\mathrm{R}}=n_{j} / \gamma_{j}$ is the plasma density in the rest frame (Asenjo et al., 2009).

An alternative form of Eq. (4) can also be found in terms of the enthalpy density $h$ (Gomberoff and Galvão, 1997).

The formalism of magnetofluid unification (Mahajan, 2003 ) is very general and allows us to study the dynamics of a charged fluid (plasma) in an electromagnetic field in a unified way, treating them as a single field. On the other hand, it also provides a general framework to study various plasma physics phenomena, taking into account relativistic temperature effects in a consistent way. In particular, the effect of relativistic temperatures on wave propagation in plasmas can be studied systematically. For the sake of simplicity, here we will consider an equal mass electron-positron plasma, where a circularly polarized electromagnetic wave propagates along a constant background magnetic field.

\subsection{Circularly polarized waves}

We consider a circularly polarized wave that propagates in the direction of a constant background magnetic field along the $z$ axis. Thus, the electric and magnetic fields are given by

$\boldsymbol{E}_{0}(z, t)=E\left[\sin \left(k_{0} z-\omega_{0} t\right) \hat{x}-\cos \left(k_{0} z-\omega_{0} t\right) \hat{y}\right]$,
$\boldsymbol{B}_{0}(z, t)=B\left[\cos \left(k_{0} z-\omega_{0} t\right) \hat{x}+\sin \left(k_{0} z-\omega_{0} t\right) \hat{y}\right]+B_{0 z} \hat{z}$,

respectively, where $k_{0}$ is the wave number and $\omega_{0}$ is the frequency of the monochromatic wave.

The amplitudes of the electric and magnetic fields can be related by Maxwell's equations as

$E=\frac{\omega_{0}}{c k_{0}} B$.

Electrons and positrons have equal constant densities $n_{\mathrm{p}}=$ $n_{\mathrm{e}}=n$, and equal constant drift velocities $\boldsymbol{v}_{z 0}=v_{z 0} \hat{z}$, so that their velocity can be written as $\boldsymbol{v}_{j}=\boldsymbol{v}_{t j}+\boldsymbol{v}_{z 0}$, where $\boldsymbol{v}_{t j}$ is transverse with respect to the background magnetic field. We will also denote their mass by $m_{\mathrm{p}}=m_{\mathrm{e}}=m$, and their charge by $q_{\mathrm{p}}=-q_{\mathrm{e}}=e$. 
In principle, we assume that electrons and positrons have constant but different temperatures, so that $f_{\mathrm{e}} \neq f_{\mathrm{p}}$. It turns out, as we will see below, that the purely transverse circularly polarized wave is an exact solution of the field equations, which is consistent with the assumption of no pressure fluctuations, that is, no fluctuations in $f$.

The particle velocities induced by the wave field are purely transverse, hence the amplitude of the circularly polarized velocity is proportional to the constant amplitude of the circularly polarized vector potential field (which can be easily shown in the Lorentz gauge, for instance), and the relativistic factor, which can be written as

$\gamma_{j}=\left(1-\frac{\boldsymbol{v}_{t j}^{2}}{c^{2}}-\frac{\boldsymbol{v}_{z 0}^{2}}{c^{2}}\right)^{-1 / 2}$,

is constant for both species.

This suggests that it is convenient to rewrite Maxwell's equations in terms of the transverse velocity $\boldsymbol{v}_{t j}$, yielding a wave equation for the vector potential

$\left(\nabla^{2}-\frac{1}{c^{2}} \frac{\partial^{2}}{\partial t^{2}}\right) \boldsymbol{A}=-\frac{4 \pi}{c} \boldsymbol{J}_{t}$,

where

$\boldsymbol{J}_{t}=\sum_{j} q_{j} n_{j} \boldsymbol{v}_{t j}$

is the transverse current. The wave Eq. (14), Poisson's equation for the electrostatic potential

$\nabla^{2} \varphi=-4 \pi \sum_{j} q_{j} n_{j}$,

the motion Eq. (4), and the continuity equation

$\frac{\partial n_{j}}{\partial t}=-\nabla \cdot\left(n_{j} \boldsymbol{v}_{j}\right)$

are the complete set of fluid equations that describe the plasma.

Defining complex transverse quantities in the form

$C_{\perp}=C_{x}+i C_{y}$

we find that

$C_{\perp}=C e^{i\left(k_{0} z-\omega_{0} t\right)}$.

Then, the particle velocity in the wave field can be written in terms of the wave magnetic field as (Asenjo et al., 2009)

$v_{j_{\perp}}=\frac{\omega^{\prime}}{f_{j} \gamma_{j} \omega^{\prime}-\Omega_{\mathrm{c} j}} \frac{q_{j} B_{\perp}}{m c k}$,

where $\Omega_{c j}=q_{j} B_{0} / m c$ and $\omega^{\prime}=\omega-k v_{z 0}$ are the gyrofrequency for both species and the Doppler shifted frequency, respectively.
Finally, this leads to the following dispersion relation for an electron-positron plasma (Asenjo et al., 2009):

$\omega^{2}-c^{2} k^{2}=\sum_{j=e, p} \omega_{\mathrm{p}}^{2}\left(\frac{\omega^{\prime}}{f_{j} \gamma_{j} \omega^{\prime}-\Omega_{\mathrm{c} j}}\right)$,

with $\omega_{\mathrm{p}}=\sqrt{4 \pi n e^{2} / m}$ the electron plasma frequency. This dispersion relation is an exact solution of the plasma equations for finite-amplitude circularly polarized propagating waves along the magnetic field in a relativistic plasma with temperature.

It is interesting to note that Eq. (21) differs from the cold plasma case (Matsukiyo and Hada, 2003) only by a factor $f_{j}$. We can understand this as follows. For single particle motion, it is possible to convert a non-relativistic result into a relativistic one by changing $m_{j} \rightarrow \gamma_{j} m_{j}$. This is possible because mass is involved in the momentum equation. However, this simple replacement is not possible for a fluid, since velocity is related nonlinearly to momentum through $\gamma_{j}$. Therefore, the average on momentum $\left\langle\boldsymbol{p}_{j}\right\rangle$ is not equivalent to $\left\langle\gamma_{j}\right\rangle\left\langle m_{j} \boldsymbol{v}_{j}\right\rangle$. We can think, though, that there is some proportionality factor between both quantities, say $f_{j}$, such that $\left\langle\boldsymbol{p}_{j}\right\rangle=f_{j}\left\langle\gamma_{j}\right\rangle\left\langle m_{j} \boldsymbol{v}_{j}\right\rangle$. Thus, it would be possible to convert a nonrelativistic fluid force equation into a relativistic one with the same prescription as for single particles, $\left\langle m_{j} \boldsymbol{v}_{j}\right\rangle \rightarrow\left\langle\boldsymbol{p}_{j}\right\rangle$, but, in order to take into account the statistics, $m_{j} \rightarrow f_{j} \gamma_{j} m_{j}$. This is exactly what is needed to go from the cold fluid dispersion relation $\left(f_{j}=\gamma_{j}=1\right.$ in Eq. 21) to Eq. (21).

For $f_{\mathrm{p}}=f_{\mathrm{e}}=1$ and no drift, the dispersion relation for a cold relativistic plasma is recovered (Matsukiyo and Hada, 2003; Gomberoff and Galvão, 1997). The same result as in Matsukiyo and Hada (2003) can be obtained from a kinetic approach (Muñoz et al., 2006), in the limit where the velocity distribution is a Dirac delta (see, e.g., Eq. (23) in Muñoz et al., 2006).

At the opposite limit of very high temperature, the transverse mode becomes a light mode $c^{2} k^{2}=\omega^{2}$, consistent with the relativistic decrease in the effective plasma frequency.

\subsection{Analysis of the dispersion relation}

In the rest of this paper, we will consider the case where both species have the same temperature, so that $f_{\mathrm{p}}=f_{\mathrm{e}}=f$. In order to study the dispersion relation Eq. (21) we will normalize all frequencies to the positron gyrofrequency $\Omega_{c p} \equiv$ $\Omega_{\mathrm{c}}$, and velocities to the speed of light $c$. It is convenient to define two adimensional parameters, related to the plasma frequency and to the wave amplitude,

$a=\frac{\omega_{\mathrm{p}}^{2}}{\Omega_{\mathrm{c}}^{2}}, \alpha=\frac{e|A|}{m c^{2}}=\frac{e\left|E_{\perp}\right|}{m c \omega}=\frac{e\left|B_{\perp}\right|}{m c^{2} k}$.

Notice that $\alpha$ corresponds to the particle transverse momentum due to the wave. 
In order to plot the dispersion relation, Eqs. (13), (20), and (21) are solved simultaneously for $\gamma_{j}, v_{j \perp}$, and $\omega$, for a given $k$, as outlined in Muñoz et al. (2006).

In Fig. 1 the dispersion relation (21) is plotted for various values of $f$, and for $a=1, \alpha=0.1$. There are two branches: an electromagnetic branch, with a lower cutoff at the effective plasma frequency, and an Alfvén branch, which has an upper cutoff in wave number, and an upper cutoff in frequency.

The solid line corresponds to the relativistic cold plasma, $f=1$. It can be seen that, as mentioned in Sect. 2.2, when the temperature increases the effective plasma frequency decreases, and the electromagnetic wave becomes a light wave. This effect can be better appreciated in Fig. 2, where the effective plasma frequency $\omega_{\mathrm{p}}^{\text {eff }}$ (given by the lower frequency cutoff for the electromagnetic branch in Fig. 1) is plotted for the same values of $f$ used in Fig. 1. $\omega_{\mathrm{p}}^{\text {eff }}$ also decreases due to the relativistic effect on the mass, and thus we plot it as a function of $\alpha$ as well. However, for high enough temperatures, the variation of the plasma frequency with wave amplitude is negligible.

Regarding the Alfvén branch, Fig. 1 shows that it starts at the origin following the usual linear dispersion relation, $\omega=v_{A} k$. In this region, $\gamma_{\mathrm{p}}, \gamma_{\mathrm{e}} \simeq 1$. Then, the Alfvén velocity can be obtained from Eq. (21) by first rewriting it in the form:

$c^{2} k^{2}=\omega^{2}-2 f \omega_{\mathrm{p}}^{2} \frac{\omega^{2}}{(f \omega)^{2}-\Omega_{\mathrm{c}}^{2}}$.

If $\omega f \ll \Omega_{\mathrm{c}}$, which is always satisfied for low enough frequencies, we find that the Alfvén velocity is given by

$v_{A}=\frac{c}{\sqrt{1+2 f \omega_{\mathrm{p}}^{2} / \Omega_{\mathrm{c}}^{2}}}$.

As wave number increases along the Alfvén branch, there is an upper frequency cutoff $\omega_{\text {crit }}$ given by

$\frac{\omega_{\text {crit }}}{\Omega_{\mathrm{c}}}=\frac{1}{f}\left[1+\left(\frac{\alpha}{f}\right)^{2 / 3}\right]^{-3 / 2}$.

Thus, when temperature increases, Alfvén waves are eventually confined to a very narrow frequency band. This can also be seen in Fig. 1.

Another interesting feature of the Alfvén branch is the existence of a maximum value for the wave number, $k_{\max }$. This upper cutoff occurs because one species (positrons, in this case) becomes ultra-relativistic, essentially moving with the speed of light in the wave field, thus, $\gamma_{\mathrm{p}}=\infty$. Electrons do not resonate with the wave, so $\gamma_{\mathrm{e}}$ is finite. In order to obtain an analytic expression for the wave number cutoff for the Alfvén branch, let us first notice that, from Eq. (20), it

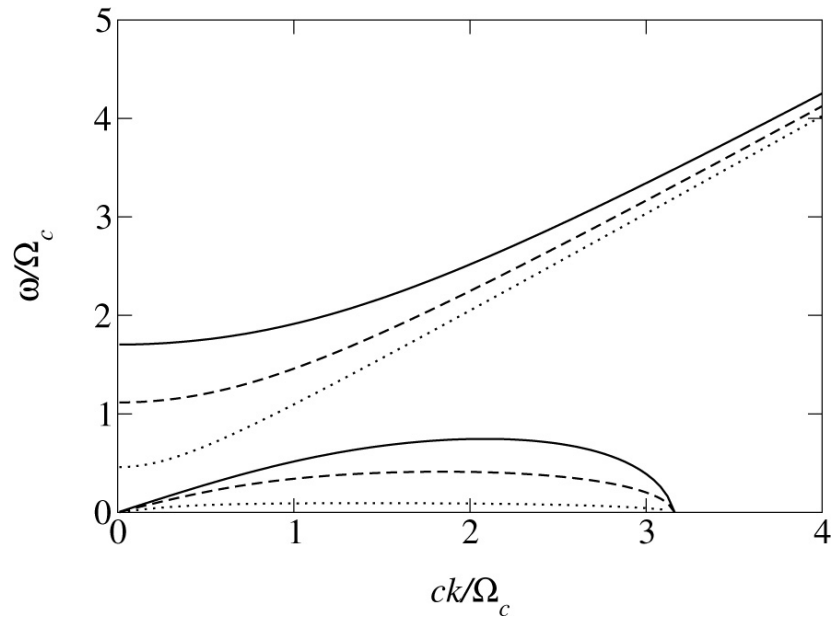

Fig. 1. Electromagnetic and Alfvén branches for the general dispersion relation, Eq. (21), for $a=1, \alpha=0.1$. Solid line: cold plasma case $(f=1)$; dashed line: $f=2$; dotted line: $f=10$.

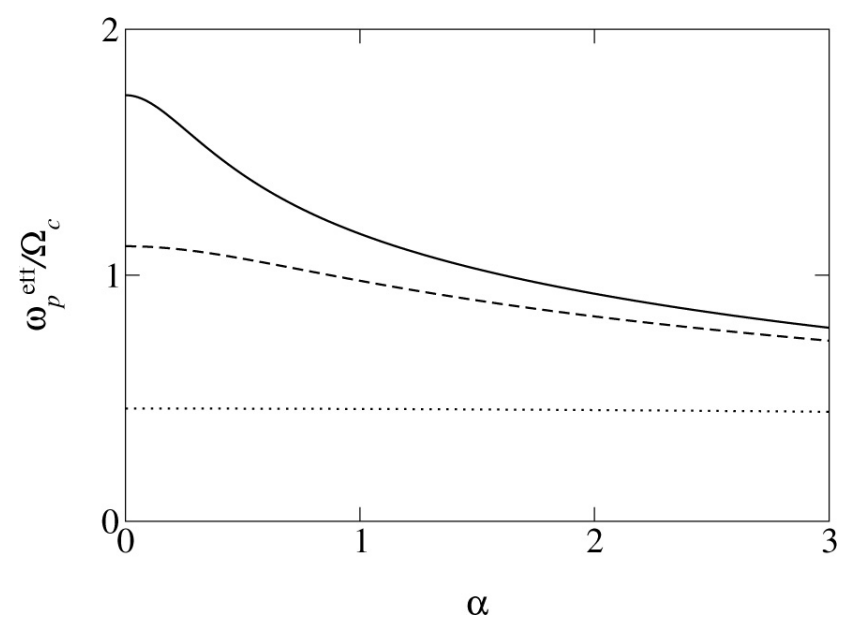

Fig. 2. Effective plasma frequency $\omega_{\mathrm{p}}^{\text {eff }}$ as a function of wave amplitude $\alpha$, for various temperatures. Solid line: cold plasma case $(f=1)$; dashed line: $f=2$; dotted line: $f=10$.

follows that

$\frac{\omega}{f \gamma_{j} \omega-\Omega_{\mathrm{c} j}}= \pm \frac{1}{\alpha} \frac{v_{\perp j}}{c}$,

where the plus (minus) sign corresponds to positrons (electrons), so the dispersion relation Eq. (21) can be written as

$c^{2} k^{2}=\omega^{2}-\frac{\omega_{\mathrm{p}}^{2}}{\alpha c}\left(v_{\perp p}-v_{\perp e}\right)$.

The cutoff occurs for $\omega=0$, and in this limit $v_{\perp p} \simeq-c$ (notice that the left-hand term in Eq. 25 is negative along the Alfvén branch for all values of $k$ ), whereas $v_{\perp e}$ becomes negligible. Thus, it follows that the maximum wave number is 
given by

$k_{\max }=\frac{\omega_{\mathrm{p}}}{c \sqrt{\alpha}}$.

This is consistent with the numerical result in Fig. 1. Notice, in particular, that $k_{\max }$ depends only on wave amplitude, not on temperature. One could argue that, if the transverse particle velocity is ultra-relativistic, then thermal velocities are not relevant.

Considering the change of sign of $\mathrm{d} \omega / \mathrm{d} k$ in the Alfvén branch, for $k<k\left(\omega_{\text {crit }}\right)$ and $k>k\left(\omega_{\text {crit }}\right)$, we will identify these regions as the normal and the anomalous zone of the Alfvén branch, respectively.

\section{Kinetic model}

In Sect. 2, an exact solution in the context of fluid theory was obtained for a circularly polarized wave propagating along a constant magnetic field in an electron-positron plasma with temperature, and its temperature dependence was studied numerically. Relativistic thermal effects produce: (a) a decrease in the effective plasma frequency (thus, waves in the electromagnetic branch can propagate for lower frequencies than in the cold case); and (b) a decrease in the upper frequency cutoff for the Alfvén branch (thus, Alfvén waves are confined to a frequency range that is narrower than in the cold case).

These results have been found in the context of a fluid model. However, effects such as Landau and cyclotron damping are absent in such a model, and thus it would be interesting to study to what extent the fluid results are modified by kinetic effects. Many works have been devoted to the study of kinetic effects on wave propagation in relativistic electronpositron plasmas, either in the absence of a magnetic field (Muñoz, 2004; Liu and Liu, 2011), to analyze the counterstreaming plasmas in pulsar environments (Verdon and Melrose, 2011), to study the effect of nonlinearities and wave damping (Chaudhary et al., 2010), to model the propagation of Bernstein waves (Gill and Heyl, 2009), etc.

In this section, we study the propagation of a largeamplitude circularly polarized electromagnetic wave in a magnetized plasma, analyzing the consistency of the results obtained in Sect. 2 with those obtained from a kinetic treatment (Muñoz et al., 2006). In effect, the same system is studied, but starting from the relativistic Vlasov equation. The corresponding kinetic dispersion relation is numerically studied for various temperatures, and results are compared with the purely fluid treatment. In our treatment, we assume that kinetic effects are only important along the propagation direction, which makes it necessary to generalize the results in Sect. 2, and to study the fluid dispersion relation Eq. (21), but in the presence of drift.

In order to gain intuition for developing the posterior kinetic analysis, in Sect. 3.1, the fluid relativistic dispersion relation of a circularly polarized electromagnetic wave propagating along a constant background magnetic field in an electron-positron plasma is solved. This corresponds to a generalization of the numerical results shown in Sect. 2 and by Asenjo et al. (2009), for the case of a plasma with constant longitudinal drift velocity. Then, the basic equations of the kinetic model are presented in Sect. 3.2. In Sect. 3.3 the dispersion relation of this wave is calculated. Then, in Sect. 3.4 the kinetic dispersion relation obtained in Sect. 3.3 is solved numerically, and results are compared with the corresponding fluid treatment. These results can also be found in Domínguez et al. (2012).

\subsection{Fluid dispersion relation with drift}

We start by defining normalized variables $x=\omega / \Omega_{\mathrm{c}}, y=$ $c k / \Omega_{\mathrm{c}}$, and $\beta=p_{z} /(m c)$, so that Eq. (21) can be written in normalized form as:

$0=y^{2}-x^{2}+\frac{\omega_{\mathrm{p}}^{2}}{\Omega_{\mathrm{c}}^{2}} \sum_{j=p, e} \frac{x \gamma_{j}-y \beta}{f\left(x \gamma_{j}-y \beta\right)-\sigma_{j}} \frac{1}{\gamma_{j}}$,

where $\sigma_{j}$ is the sign of the charge of species $j$, so that $\sigma_{\mathrm{p}}=1$, and $\sigma_{\mathrm{e}}=-1$.

In order to solve the fluid dispersion relation given by Eq. (28), the roots of the following quartic equation for $\gamma_{j}$ must be found:

$$
\begin{aligned}
0 & =\gamma_{j}^{4} f^{2}-\gamma_{j}^{3} \frac{2 f}{x}\left(y \beta f+\sigma_{j}\right) \\
& +\gamma_{j}^{2}\left[\frac{\left(y \beta f+\sigma_{j}\right)^{2}}{x^{2}}-\left(1+\beta^{2}\right) f^{2}-\alpha^{2}\right] \\
& +\gamma_{j} \frac{2}{x}\left[\left(1+\beta^{2}\right) f\left(y \beta f+\sigma_{j}\right)+y \beta \alpha^{2}\right] \\
& -\frac{1}{x^{2}}\left[\left(1+\beta^{2}\right)\left(y \beta+\sigma_{j}\right)^{2}+y^{2} \beta^{2} \alpha^{2}\right] .
\end{aligned}
$$

Equation (29) is the generalization of Eq. (15) in Muñoz et al. (2006) for $f \neq 1$ and $\beta \neq 0$. In Sect. 2 the case $f \neq 1$ was solved (Asenjo et al., 2009). We now consider the general case given by Eq. (29), to solve the dispersion relation given by Eq. (28) for both nonzero temperatures $(f \neq 1)$ and nonzero drift velocities $(\beta \neq 0)$.

To do this, we first choose fixed values for $y, \beta, f$ and $\alpha$, and a trial value for $x$. Equation (29) is then solved for $\gamma_{\mathrm{p}}$ and $\gamma_{\mathrm{e}}$. Only solutions $\gamma_{j} \geq 1$ are physically acceptable. This yields several possible pairs $\left(\gamma_{\mathrm{p}}, \gamma_{\mathrm{e}}\right)$. Each possible pair corresponds to a different branch of the dispersion relation. When a pair is chosen, then the right-hand size of Eq. (28) can be evaluated. However, it turns out that, in general, the number of acceptable solutions of Eq. (29) for $\gamma_{\mathrm{e}}$ and $\gamma_{\mathrm{p}}$ is greater than 2, and this number changes for each value of $\beta$. This issue complicates the choice of $\gamma_{j}$. 
This is illustrated in Fig. 3. For given values of $y$, $\alpha$, and $f$, we solve Eq. (29) for a certain value of $x$ and $\beta$, obtaining four roots, $\gamma_{j}^{(s)}$. We sort them such that $\operatorname{Re} \gamma_{j}^{(1)} \leq \operatorname{Re} \gamma_{j}^{(2)} \leq \operatorname{Re} \gamma_{j}^{(3)} \leq \operatorname{Re} \gamma_{j}^{(4)}$. Figure 3 shows, for a certain range of values of $x$ and $\beta$, the regions where the sth branch for positrons, $\gamma_{\mathrm{p}}^{(s)}$, is physically acceptable, that is, real and greater than 1 .

Figure 3 shows that for $x>0.8$, approximately, there is only one possible choice, $\gamma_{\mathrm{p}}^{(3)}$. However, for lower values of the frequency, there is a range of values of $\beta$ where more than one choice of $\gamma_{p}$ is possible. For instance, for $x=0.400087, y=0.3$ and $\beta=4.4$, the three possible values are $\gamma_{\mathrm{p}}^{(1)}=4.12392, \gamma_{\mathrm{p}}^{(2)}=5.72992$, and $\gamma_{\mathrm{p}}^{(3)}=5.86722$.

With a similar analysis in the case of $\gamma_{\mathrm{e}}$, now we choose one of the possible branches for $\gamma_{\mathrm{p}}$, and one of the branches for $\gamma_{\mathrm{e}}$, and we can calculate the right-hand side of Eq. (28) by solving for the value of $x$ for which it is zero. Notice that it could still be the case that a chosen pair $\left(\gamma_{\mathrm{p}}, \gamma_{\mathrm{e}}\right)$ yields an Eq. (28) with no real solutions for $x$. It turns out that there is only one choice of the $\left(\gamma_{\mathrm{e}}, \gamma_{\mathrm{p}}\right)$ pair for which a real root of Eq. (28) can be found.

In Fig. 4 we plot the solutions of the dispersion relation (28), obtained by the method outlined above. The solid line, $\beta=0$, corresponds to the solution previously shown in Fig. 1 (Asenjo et al., 2009), which shows that our treatment leads to the correct limit in the absence of drift.

Notice that the Alfvén branch is much more sensitive to variations in drift than the electromagnetic branch. For small values of the frequency, it follows the usual linear dispersion relation, $\omega \simeq v_{A} k$, with $v_{A}$ the Alfvén speed. $v_{A}$ can be calculated by noting that, in this region, $\gamma_{\perp p}, \gamma_{\perp e} \sim 1+\beta^{2}$, and by setting $\omega \ll \Omega_{\mathrm{c}}$ in Eq. (21). This yields

$\frac{v_{A}}{c}=\frac{2 f \beta \sqrt{1+\beta^{2}}+v_{A 0} \sqrt{\left(1+\beta^{2}\right) v_{A 0}^{2}+2 f \sqrt{1+\beta^{2}}}}{v_{A 0}^{2} \sqrt{1+\beta^{2}}+2 f\left(1+\beta^{2}\right)}$,

where

$v_{A 0}=\frac{\Omega_{\mathrm{c}}}{\omega_{\mathrm{p}}}$

is the Alfvén velocity in the nonrelativistic cold fluid case.

For $\beta=0$, the result in Eq. (23) is recovered, which we can rewrite as

$\frac{v_{A}}{c}=\frac{v_{A 0}}{\sqrt{v_{A 0}^{2}+2 f}}$.

In Fig. 5 we plot the Alfvén velocity. In principle, $v_{A}$ depends both on the drift velocity and the temperature. For illustration purposes, in Fig. 5 we have first taken a fixed temperature (Fig. 5a), and then a fixed drift velocity (Fig. 5b). Qualitatively similar results are obtained for other choices of $f$ and $\beta$. What is important to notice is that the drift velocity and the temperature have the opposite effect on the Alfvén speed: $v_{A}$ increases with $\beta$, and decreases with $f$.
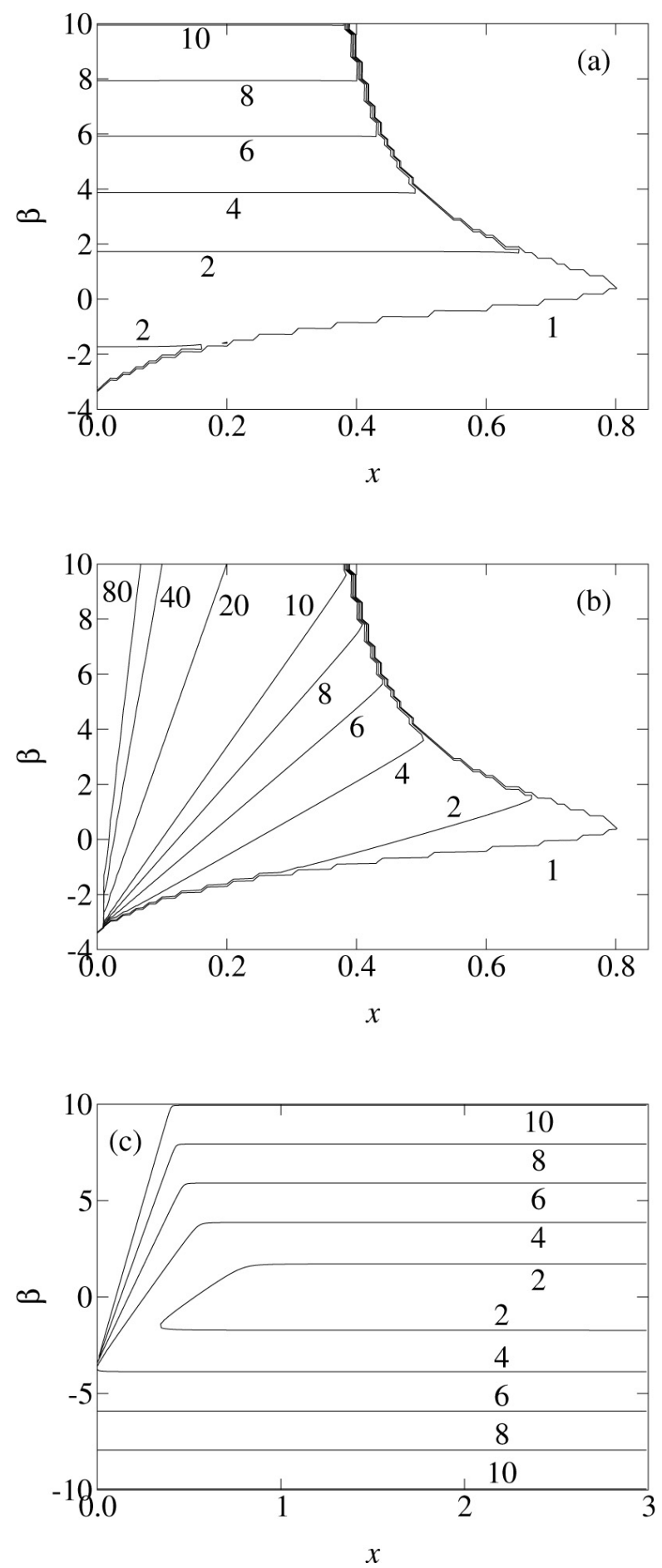

Fig. 3. Isocontours of real solutions of Eq. (29) as a function of normalized drift $\beta$ and normalized frequency $x$, for positrons. Each panel corresponds to a different branch, $\gamma_{\mathrm{p}}^{(s)}$, for the roots of Eq. (29). A few contour levels are shown. Physically acceptable solutions are in the regions where $\gamma(x, \beta)$ is real and greater than 1. Fixed values $y=0.3, \alpha=0.1$, and $f=1$ were taken. (a) $s=1$, (b) $s=2$, and (c) $s=3$. 


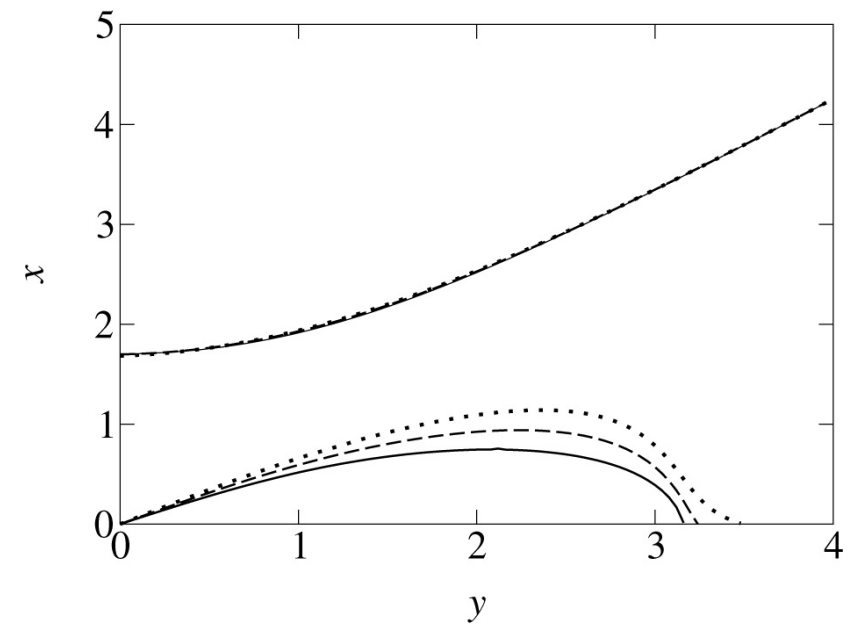

Fig. 4. Solutions of the dispersion relation Eq. (21) for $f=1$, $\alpha=0.1, v_{A 0}=1$ (see Eq. (31) below), and three values of $\beta: \beta=0$ (solid line), $\beta=0.1$ (dashed line), $\beta=0.2$ (dotted line).

\subsection{Kinetic model}

In our treatment, we consider kinetic effects only along a background magnetic field $B_{0 z} \hat{z}$, and a cold fluid model in the perpendicular direction (Muñoz and Gomberoff, 2002; Muñoz, 2004).

As in Sect. 2, the electromagnetic fields are described by Eqs. (14)-(16), whereas the fluid is described by the continuity equation (17) and, in the transverse direction, the motion equation (4) with zero temperature:

$\left(\frac{\partial}{\partial t}+\boldsymbol{v}_{j} \cdot \nabla\right)\left(\gamma \boldsymbol{v}_{j}\right)=\frac{q_{j}}{m_{j}}\left(\boldsymbol{E}+\frac{1}{c} \boldsymbol{v}_{j} \times \boldsymbol{B}\right)$.

For the longitudinal direction, we consider the onedimensional Vlasov equation:

$\frac{\partial g_{j}}{\partial t}+\boldsymbol{v} \cdot \nabla g_{j}+\frac{q_{j}}{m_{j}}\left(\boldsymbol{E}+\frac{1}{c} \boldsymbol{v} \times \boldsymbol{B}\right) \cdot \nabla_{v} g_{j}=0$,

where $g_{j}$ is the velocity distribution function for species $j$, normalized so that

$\int \mathrm{d} \boldsymbol{v} g_{j}(\boldsymbol{r}, \boldsymbol{v}, t)=n_{j 0}$,

with $n_{j 0}$ as the equilibrium unperturbed density for species $j$, and Poisson Eq. (16).

Thus, the total density and transverse current are given by

$n_{j}=\sum_{j} \int \mathrm{d} \boldsymbol{v} g_{j}(\boldsymbol{r}, \boldsymbol{v}, t)$,

$\boldsymbol{J}_{t}=\sum_{j} q_{j} \int \mathrm{d} \boldsymbol{v} g_{j}(\boldsymbol{r}, \boldsymbol{v}, t) \boldsymbol{v}_{j t}$,

respectively.
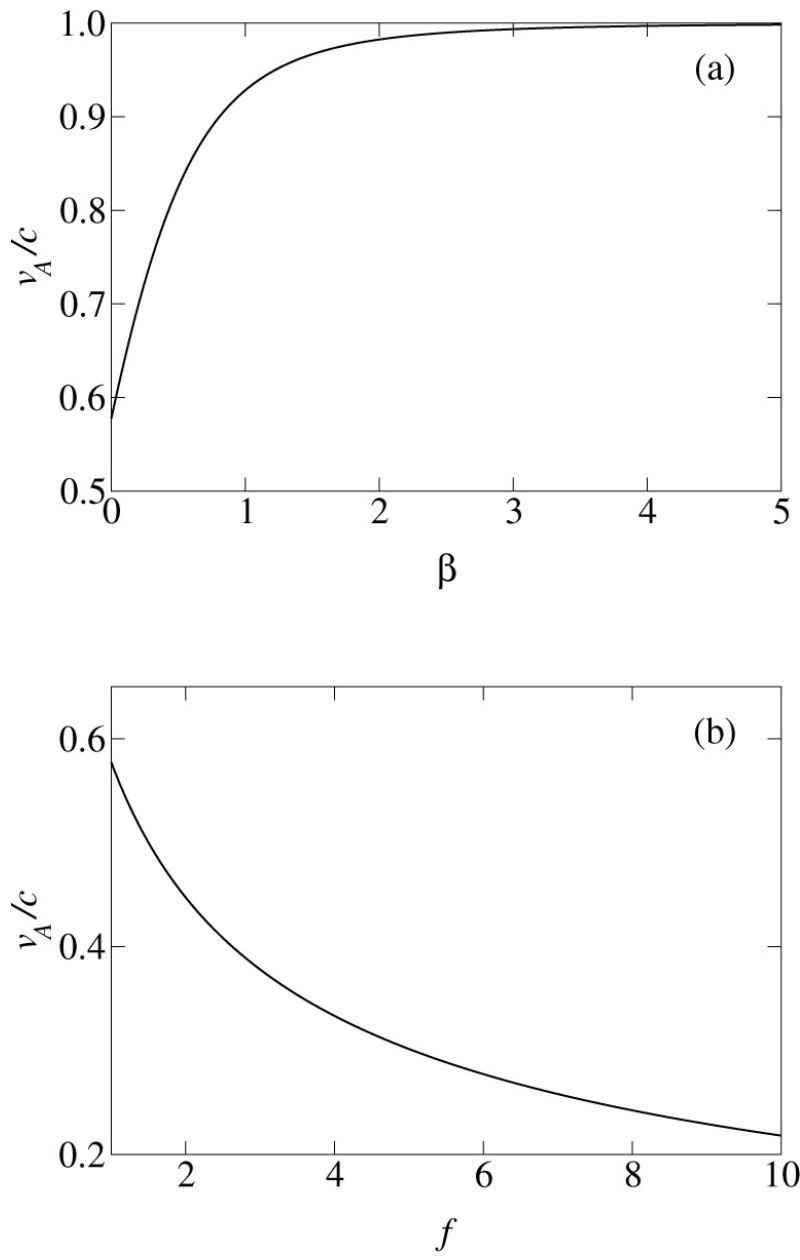

Fig. 5. Alfvén velocity, Eq. (30), for $\alpha=0.1, v_{A 0}=1$. (a) For fixed temperature, $f=1$, as a function of the drift velocity $\beta$. (b) For fixed drift velocity, $\beta=0$, as a function of temperature, parameterized by $f$.

\subsection{Kinetic dispersion relation}

As in Sect. 2, we now consider an electromagnetic wave propagating in the electron-positron plasma. In the absence of the wave, the plasma is considered to have no electric field, a uniform background magnetic field $B_{0 z} \hat{z}$, equal densities for each species $\left(n_{e 0}=n_{p 0}=n_{0}\right)$, and equal drifts for each $\operatorname{species}\left(\boldsymbol{v}_{j 0}=v_{z 0} \hat{z}\right)$.

We consider a circularly polarized wave that propagates along the $\hat{z}$ axis, whose electric and magnetic fields are given by Eqs. (10) and (11).

In terms of the transverse quantities Eq. (18), Maxwell and Vlasov equations yield:

$\left(\nabla^{2}-\frac{1}{c^{2}} \frac{\partial^{2}}{\partial t^{2}}\right) A_{\perp}=-\frac{4 \pi}{m c} \sum_{j} q_{j} \int \mathrm{d} \boldsymbol{p} v_{j \perp} g_{j}(\boldsymbol{x}, \boldsymbol{v}, t)$. 
On the other hand, in order to introduce kinetic effects, we consider the following form of the distribution function:

$g_{j}=n_{0} \tilde{g}_{j}\left(z, p_{z}, t\right) \delta\left(p_{x}-p_{x}^{f}\right) \delta\left(p_{y}-p_{y}^{f}\right)$,

where $\boldsymbol{p}^{f}$ is the transverse particle momentum obtained with the cold fluid theory. In the absence of the wave, the adimensional distribution function in Eq. (38) must satisfy $\tilde{g}_{e 0}=$ $\tilde{g}_{p 0}$, so that densities are equal.

Then, Eq. (37) can be written in the form:

$$
\left(\nabla^{2}-\frac{1}{c^{2}} \frac{\partial^{2}}{\partial t^{2}}\right) A_{\perp}=-\frac{4 \pi n_{0}}{m c} \sum_{j} q_{j} \int \mathrm{d} p_{\perp} v_{j \perp} \tilde{g}_{j}\left(z, p_{z}, t\right)
$$

Note that we need to know $v_{j \perp}$ in order to be able to solve the dispersion relation. To do this, we consider that in the direction perpendicular to the magnetic field, the equations governing the system are the fluid equations, so that the transverse velocity is given by Eq. (20). In Domínguez et al. (2012) it is shown that the following dispersion relation is obtained:

$0=k^{2} c^{2}-\omega^{2}+\omega_{\mathrm{p}}^{2} \sum_{j} \int \mathrm{d} p_{z} \frac{\omega^{\prime}}{\omega^{\prime} \gamma_{j}-\Omega_{c j}} \tilde{g}_{j 0}$.

\subsection{Numerical solution of the kinetic dispersion relation}

We now solve the kinetic dispersion relation Eq. (40). First, it is convenient to write it in normalized form:

$y^{2}-x^{2}+\frac{\omega_{\mathrm{p}}^{2}}{\Omega_{\mathrm{c}}^{2}} \sum_{j=p, e} \int \mathrm{d} \beta \frac{x \gamma_{j}-\beta y}{x \gamma_{j}-\beta y-\sigma_{j}} \frac{\tilde{g}_{j 0}}{\gamma_{j}}=0$.

There are two main issues to consider at this point. One is the choice of the distribution function, and the other is the choice of $\gamma_{j}$.

Regarding the distribution function, since we are interested in comparing the kinetic dispersion relation with the results in Sect. 2, which include the effects of relativistic temperature, we take $\tilde{g}_{p 0}=\tilde{g}_{e 0} \equiv \tilde{g}_{0}$ as a Maxwell-BoltzmannJüttner distribution:

$\tilde{g}_{0}(\beta, \mu)=\frac{1}{2 m c K_{1}(\mu)} e^{-\mu\left(1+\beta^{2}\right)^{1 / 2}}$.

Thus, in the kinetic treatment, thermal information is contained in the normalized inverse temperature parameter $\mu$. In the fluid treatment described in Sect. 2, thermal information is contained in the parameter $f$.

Once the distribution function is chosen, the numerical strategy is analogous to the one outlined in Sect. 3.1, except that an integration along the $\beta$ axis must be done. This means that, for each trial frequency, the corresponding values of $\gamma(\beta)$ must be found. Since, in the transverse direction, we consider a cold fluid model, the problem of finding $\gamma$ is equivalent to the one discussed in Sect. 3.1 for a cold fluid with a given longitudinal drift $\beta$. Thus, the equation to solve in order to find $\gamma$ is Eq. (29), with $f=1$. It must be solved for each point in the $\beta$ axis, and once the integration is complete, the right-hand size of Eq. (40) can be evaluated. Thus, rather than finding a value of, say, $\gamma_{\mathrm{e}}$ to generate the electromagnetic branch in the fluid dispersion relation, the proper infinite set of values $\gamma_{\mathrm{e}}(\beta)$ must be found.

If only two physically acceptable values of $\gamma_{j}$ were obtained for each value of $\beta$, then the problem would still be easy, since one of them would correspond to the electromagnetic branch, and the other to the Alfvén branch, and there would be no ambiguity in the calculation of the integral. However, as shown in Sect. 3.1, several values of $\gamma_{j}$ are possible, both for electrons and positrons. Moreover, the problem is now more complicated, since in the kinetic case, there is an integral over $\beta$, thus a choice of branches for $\gamma_{j}$ must be made at each point of the integration path, and since the number of valid values of $\gamma_{j}$ changes with $\beta$ (see Fig. 3), there is no guarantee that a choice for a given $\beta$ is valid along the complete integration path. In fact, we have found that this is not the case in general. By studying several families of parameters, we concluded that it is very difficult to find a general, reliable algorithm to automate the choice, and some trial and error is still involved in the results presented here.

The rest of the numerical procedure is equivalent to the fluid case: once a proper choice of $\gamma_{j}(x, \beta)$ is made, it is used to find a value of $x$ such that Eq. (41) is satisfied, and the process is repeated for a different value of $x$.

Figure 6 shows the solution of the dispersion relation Eq. (21) for three different temperatures. This figure should be compared with Fig. 1, where three temperatures corresponding to $f=1,2$ and 10 were considered.

We should first notice that, in the Alfvén branch, which starts at the origin, there is a small-frequency region where no modes are found (in this case $y \sim 2$ ). This is a result of the technical issues regarding the choice of $\gamma_{j}$ at each point of the integration path, and illustrated in Fig. 3. On the other hand, the electromagnetic branch (for all wave numbers) and the Alfvén branch near the origin (for small values of $x=\omega / \Omega_{\mathrm{c}}$ and $y=c k / \Omega_{\mathrm{c}}$ ) can always be obtained without ambiguities in the choice of $\gamma_{j}$.

Thus, we will focus the remainder of the discussion on the electromagnetic branch and the region of the Alfvén branch near the origin. Solving the numerical issues near the maximum frequency in the Alfvén branch may involve either improving the numerical algorithms, or improving on the cold model for the transverse motion (Eq. 38). In fact, preliminary results from PIC simulations (Sect. 5) suggest that the Alfvén branch is damped in the anomalous zone, and we plan to study this elsewhere.

The first result to notice is that, indeed, the same branches as in the fluid case are found, an electromagnetic and an Alfvén branch. For very low temperatures, the cold result is recovered (see solid line in Fig. 4). When the temperature is increased ( $\mu$ decreases), it is observed that the effect of the 


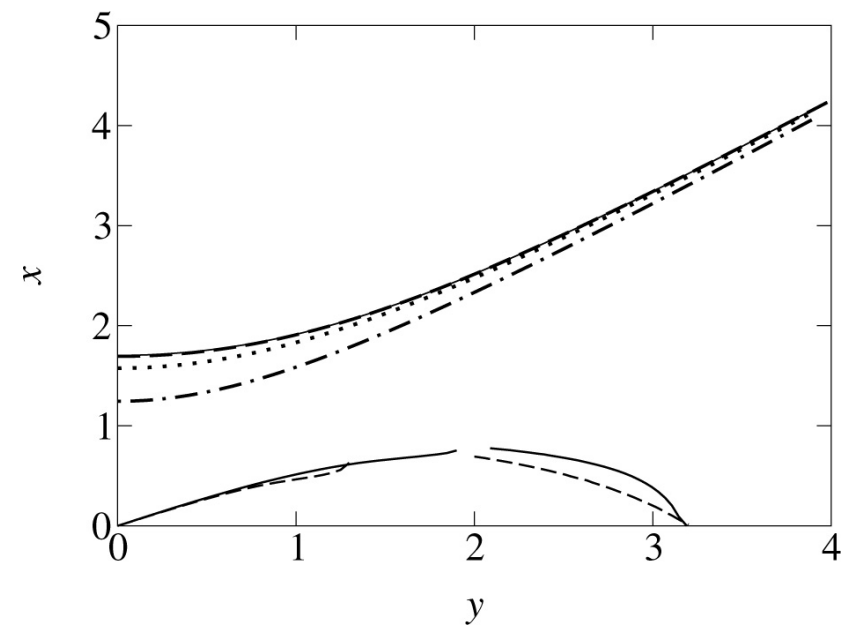

Fig. 6. Solutions of the dispersion relation Eq. (40) for $\alpha=0.1$ and $v_{A 0}=1$. Solid line $\mu=700(f \simeq 1)$; dashed line $\mu=50(f \simeq 1.1)$; dotted line $\mu=3(f \simeq 2)$; dot dashed line $\mu=0.4(f \simeq 10)$. At the scale of the figure, the dashed and solid lines for the electromagnetic branch cannot be distinguished. Also, the dotted and dot dashed lines for the Alfvén branch cannot be seen; this occurs because, for high temperatures, the Alfvén branch disappears, as discussed later in Fig. 8.

temperature is to decrease the effective plasma frequency, and to decrease the upper frequency cutoff for the Alfvén branch. It is interesting to observe that this is qualitatively equivalent to what is obtained in the fluid theory.

However, several quantitative differences are found. First, in the kinetic model, the effective plasma frequency is less sensitive to variations in temperature than in the fluid model. This is shown in Fig. 7, where $\omega_{\mathrm{p}}^{\text {eff }}$ (solution of the electromagnetic branch, Eq. 41 with $y=0$ ) is plotted as a function of inverse normalized temperature $\mu$, for both models. For any given temperature, the plasma frequency is always higher in the kinetic case than in the fluid case.

Regarding the Alfvén branch, analytical results can be obtained from Eq. (40), which is consistent with the numerical procedures that yield Fig. 6. Taking $\omega \ll \Omega_{\text {c }}$ in Eq. (40) yields the Alfvén velocity

$\frac{v_{A}}{c}=\sqrt{\frac{v_{A 0}^{2}-I_{1}(\mu)}{v_{A 0}^{2}+I_{2}(\mu)}}$,

where

$$
\begin{aligned}
& I_{1}(\mu)=2 \int \mathrm{d} \beta \frac{g_{0}(\beta, \mu) \beta^{2}}{\sqrt{1+\beta^{2}}}, \\
& I_{2}(\mu)=2 \int \mathrm{d} \beta g_{0}(\beta, \mu) \sqrt{1+\beta^{2}} .
\end{aligned}
$$

The kinetic result Eq. (42), as well as the fluid result, Eq. (23), are plotted as a function of $\mu$ in Fig. 8, for various selected values of $v_{A 0}$.

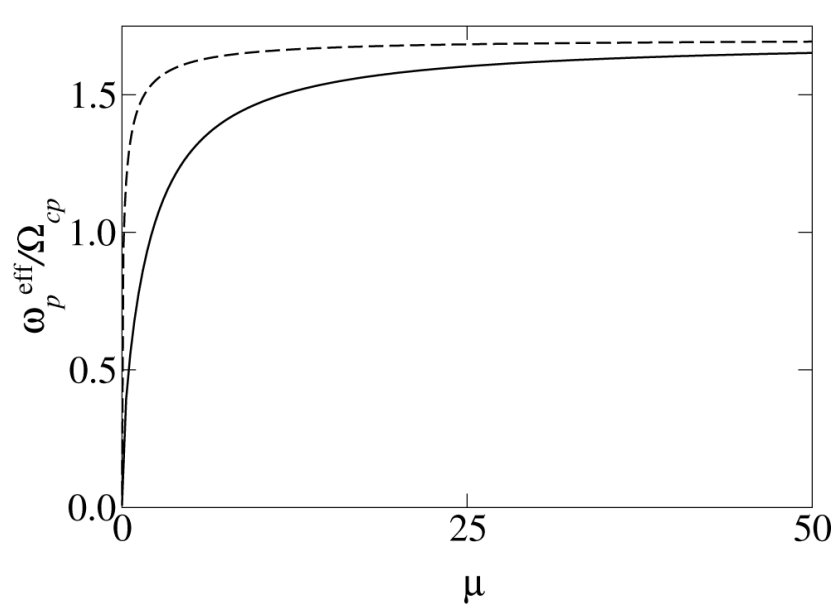

Fig. 7. Effective plasma frequency as a function of inverse normalized temperature $\mu$. Solid line: fluid model. Dashed line: kinetic model.
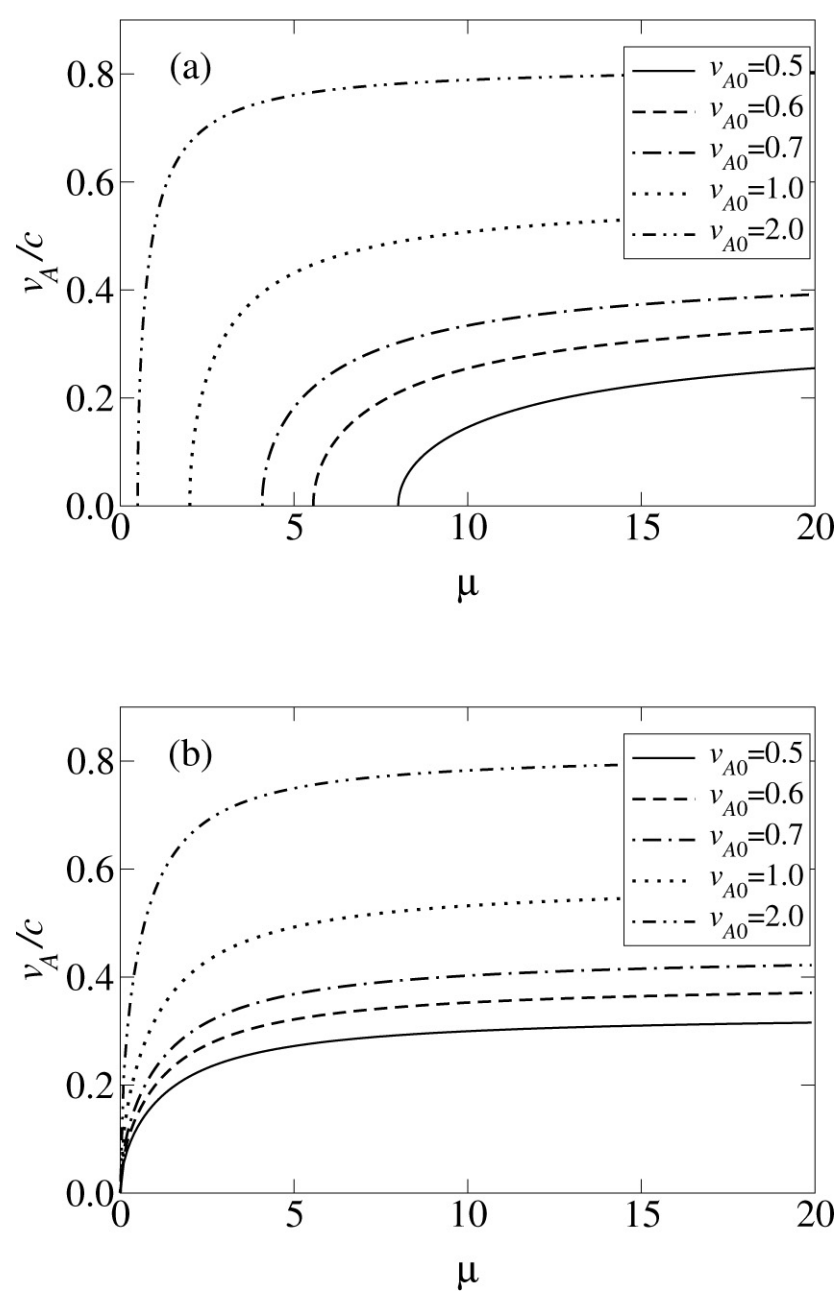

Fig. 8. Alfvén speed as a function of inverse normalized temperature of $\mu$. (a) Eq. (42) and (b) Eq. (30) $(\beta=0)$, for various values of $v_{A 0}$. 
We notice that increasing the temperature has the effect of decreasing the Alfvén speed (Fig. 8a). The same qualitative behavior occurs in the fluid case (Fig. 8b). However, in the kinetic treatment, unlike the fluid case, the Alfvén branch is suppressed for high temperatures. The threshold temperature (proportional to $\mu^{-1}$ ), below which the Alfvén branch exists, is larger if $v_{A 0}$ increases. That is, if, say, the density is constant, the Alfvén branch exists for a larger range of temperatures when the background magnetic field is increased. The threshold can be obtained from Eq. (42), by noting that it satisfies

$v_{A 0}^{2}-I_{1}\left(\mu_{\min }\right)=0$.

Figure 9 shows the value of $\mu_{\min }$ for various values of $v_{A 0}$. If the density is fixed, Fig. 9 shows that Alfvén waves can propagate for larger values of the temperature ( $\mu_{\min }$ is smaller) if the background magnetic field is increased ( $v_{A 0}$ increases).

Figure 8 also shows that, for a given value of $v_{A 0}$ (fixed amplitude of the background magnetic field, for instance), the maximum Alfvén speed is obtained in the cold limit.

The result obtained in Eq. (42) is fully consistent with the fluid model in the cold limit. In fact, if $\mu \rightarrow \infty$ in Eqs. (43) and (44), then $I_{1} \rightarrow 0$ and $I_{2} \rightarrow 2$, thus recovering Eq. (23).

In Fig. 10 we plot the maximum value of the Alfvén speed as a function of $v_{A 0}$ as given by the kinetic model (Eq. 42 with a very large value of $\mu$ ), and by the fluid model (Eq. 23, with $f=1$ ).

The fact that both results match shows that, in spite of the technical issues discussed above regarding the proper choice of $\gamma$ at each point in the integration path, the kinetic model presented here still yields the fluid result in the proper limit.

\section{Parametric decays}

\subsection{Parametric decays}

In this section we will consider the parametric perturbations of finite-amplitude circularly polarized electromagnetic waves in a relativistic electron-positron thermal plasma, which were found in Sect. 2. Although simple, this analysis will allow us to study in detail the effect of relativistic temperatures on wave propagation and its decay in relativistic hot plasmas. These decays have been studied for electron-positron plasmas in the weakly relativistic limit for linear polarization (Gomberoff et al., 1997; Muñoz and Gomberoff, 2000), and in the magnetized (Muñoz and Gomberoff, 1998b) and nonmagnetized cases (Muñoz and Gomberoff, 1998a) with circular polarization. Similarly, decays of circularly polarized Alfvén waves have been studied (Matsukiyo and Hada, 2003), also in the weakly relativistic regime. On the other hand, the effect of relativistic temperatures has not been considered in these studies.

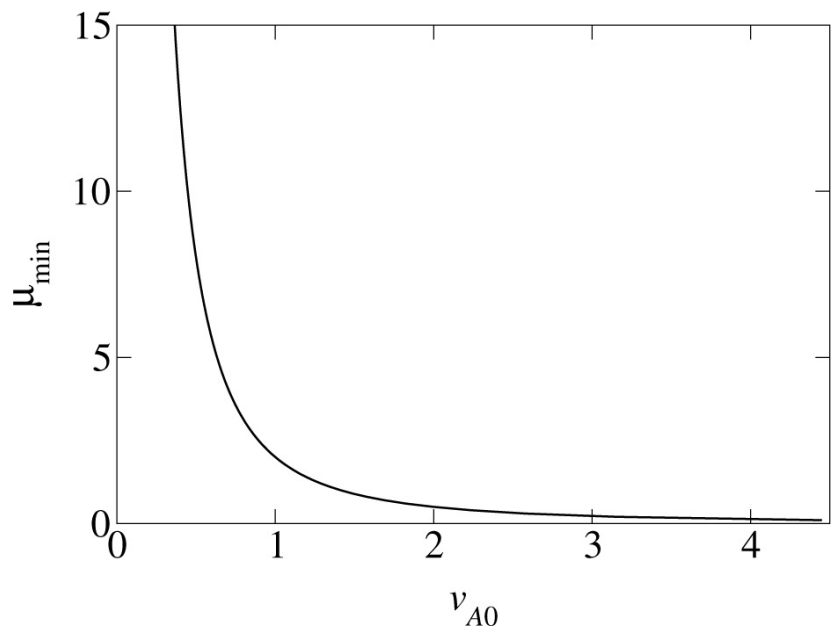

Fig. 9. Minimum value of $\mu$ as a function of $v_{A 0}$.

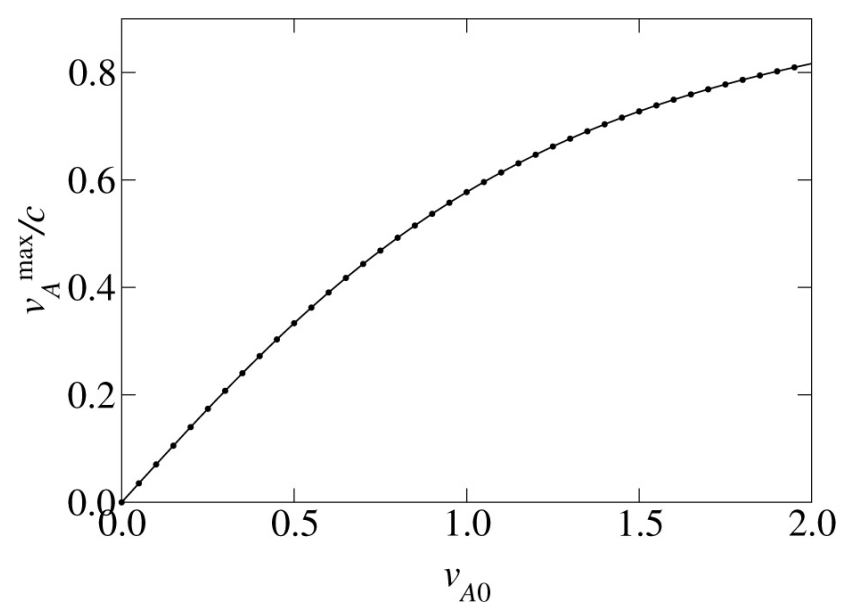

Fig. 10. Maximum Alfvén speed as a function of $v_{A 0}$, obtained from solving the kinetic dispersion relation Eq. (40) for very low temperature (solid line), and from the fluid model, Eq. (23) (dotted).

In Sect. 4.3, the dispersion relation for the parametric decays is presented. In Sect. 4.4, this dispersion relation is solved numerically and several features are discussed.

\subsection{Exact solution}

In the parametric decay analysis, the exact circularly polarized electromagnetic wave found in Sect. 2 corresponds to the zeroth-order solution. Thus, zeroth-order fields are given by Eqs. (10) and (11), with the corresponding quiver velocities given by (see Eq. 20)

$v_{\perp 0 j}=\left(\frac{\omega_{0}}{f_{0 j} \gamma_{0 j} \omega_{0}-\Omega_{\mathrm{c} j}}\right) \frac{q_{j} B_{\perp 0}}{m c k_{0}}$,

which can be rewritten as

$v_{\perp 0 j}=\alpha_{j} \eta_{j} c e^{i\left(k_{0} z-\omega_{0} t\right)}$, 
with

$\alpha_{j}=\sigma_{j} \alpha$,

$\eta_{j}=\frac{\omega_{0}}{f_{0 j} \gamma_{0 j} \omega_{0}-\Omega_{c j}}$.

The dispersion relation for the pump wave is (see Eq. 21)

$\omega_{0}^{2}-c^{2} k_{0}^{2}=\sum_{j} \omega_{\mathrm{p}}^{2}\left(\frac{\omega_{0}}{f_{0 j} \gamma_{0 j} \omega_{0}-\Omega_{\mathrm{c} j}}\right)$.

\subsection{Parametric decays}

Now we introduce small perturbations on the zeroth-order solution given by the pump wave as described in Sect. 4.2. Thus, a physical quantity $C$ is written in the form $C=C_{0}+$ $\delta C$, where $C_{0}$ corresponds to the pump wave solution, and $\delta C \ll C$.

To first order in the perturbed quantities, we obtain the following set of perturbed equations (López et al., 2012)

$$
\begin{aligned}
& f_{0}\left(\frac{\partial}{\partial t}+\boldsymbol{v}_{0 j} \cdot \nabla\right)\left(\gamma_{0 j} \delta \boldsymbol{v}_{j}+\delta \gamma_{j} \boldsymbol{v}_{0 j}\right) \\
& +f_{0}\left(\delta \boldsymbol{v}_{j} \cdot \nabla\right)\left(\gamma_{0 j} \boldsymbol{v}_{0 j}\right)+\gamma_{0 j}\left(\frac{\partial}{\partial t}+\boldsymbol{v}_{0 j} \cdot \boldsymbol{\nabla}\right)\left(\boldsymbol{v}_{0 j} \delta f_{j}\right) \\
& =\frac{q_{j}}{m}\left(\delta \boldsymbol{E}+\frac{1}{c} \boldsymbol{v}_{0 j} \times \delta \boldsymbol{B}\right)+\frac{q_{j}}{m c} \delta \boldsymbol{v}_{j} \times \boldsymbol{B}_{0}-\frac{1}{m n_{0}} \nabla \delta p_{j}, \\
& \frac{\partial \delta p_{j}}{\partial t}=m c^{2} n_{0 j}^{\mathrm{R}} \frac{\partial \delta f_{j}}{\partial t} \\
& \frac{\partial \delta n_{j}}{\partial t}+n_{0} \boldsymbol{\nabla} \cdot \delta \boldsymbol{v}_{j}=0, \\
& \nabla \cdot \delta \boldsymbol{E}=4 \pi \sum_{j} q_{j} \delta n_{j},
\end{aligned}
$$

and

$\boldsymbol{\nabla} \times \delta \boldsymbol{B}=\frac{4 \pi}{c} \sum_{j} q_{j}\left(\delta n_{j} \boldsymbol{v}_{j}+n_{j} \delta \boldsymbol{v}_{j}\right)+\frac{1}{c} \frac{\partial \delta \boldsymbol{E}}{\partial t}$.

We now introduce perturbations on the transverse quantities (18) such that $\delta C_{\perp} \ll C_{\perp}, \delta C_{z} \ll C_{z}$. We also assume that longitudinal and transverse perturbations have the form

$\delta C_{z}=\operatorname{Re}\left[\tilde{C} e^{i(k z-\omega t)}\right]=\frac{1}{2}\left(\tilde{C} e^{i(k z-\omega t)}+\tilde{C}^{*} e^{-i\left(k^{*} z-\omega^{*} t\right)}\right)$,

and

$\delta C=c_{+} e^{i\left(k_{+} z-\omega_{+} t\right)}+c_{-} e^{i\left(k_{-} z-\omega_{-} t\right)}$,

respectively, where $k_{+}=k_{0}+k, k_{-}=k_{0}-k^{*}, \omega_{+}=\omega_{0}+\omega$ and $\omega_{-}=\omega_{0}-\omega^{*}$. With these definitions, and after a lengthy but straightforward calculation (see López et al., 2012 for details), we obtain a set of equations for the variables $\mathbf{r}=$ $\left(v_{+e}, v_{+p}, v_{-e}^{*}, v_{-p}^{*}, \tilde{v}_{\mathrm{e}}, \tilde{v}_{\mathrm{p}}, b_{+}, b_{-}^{*}\right)$, which can be put in matrix form as

$\mathbb{A} \mathbf{r}=0$, so that the dispersion relation is given by

$F(k, \omega)=\operatorname{det}(\mathbb{A})=0$.

The matrix elements $\mathbb{A}_{i j}$ are given explicitly in López et al. (2012). This turns out to yield a polynomial equation of degree 12 for $\omega(k)$.

In the cold, weakly relativistic case, we recover the results previously found by Muñoz and Gomberoff (1998b).

When there is no pump wave, $\alpha=0$, Eq. (57) yields the normal modes that propagate in the plasma. At this limit, it is not difficult to show that the dispersion relation (57) becomes

$F_{S D}(k, \omega)=D_{+} D_{-} S L=0$,

where

$S=-\omega^{2}+v_{\mathrm{s}}^{2} k^{2}$,

$L=\frac{2 \omega_{\mathrm{p}}^{2}}{f_{0}}-\omega^{2}+v_{\mathrm{s}}^{2} k^{2}$,

and

$$
\begin{aligned}
& D_{+}=\omega_{+}^{2}-c^{2} k_{+}^{2}-\frac{\omega_{\mathrm{p}}^{2}}{f_{0}}\left(\frac{\omega_{+}}{\omega_{+}-\frac{\Omega_{\mathrm{c}}}{f_{0}}}+\frac{\omega_{+}}{\omega_{+}+\frac{\Omega_{\mathrm{c}}}{f_{0}}}\right), \\
& D_{-}=\omega_{-}^{* 2}-c^{2}{k_{-}^{*}}^{2}-\frac{\omega_{\mathrm{p}}^{2}}{f_{0}}\left(\frac{\omega_{-}^{*}}{\omega_{-}^{*}-\frac{\Omega_{\mathrm{c}}}{f_{0}}}+\frac{\omega_{-}^{*}}{\omega_{-}^{*}+\frac{\Omega_{\mathrm{c}}}{f_{0}}}\right),
\end{aligned}
$$

and where we have defined

$$
v_{\mathrm{s}}=c \sqrt{\frac{1}{f_{0}} \frac{f^{\prime}\left(\mu_{0}\right) \mu_{0}}{1+f^{\prime}\left(\mu_{0}\right) \mu_{0}^{2}}} .
$$

Equation (59) suggests that $v_{\mathrm{s}}$ can be defined as an effective sound velocity.

In Fig. 11 we compare the effective sound velocity as given by Eq. (63) with the classical sound velocity, $v_{\mathrm{s}}=$ $\sqrt{k_{B} T / m}$. For low temperatures both results are equivalent, whereas for high temperatures the effective sound velocity tends to $c / \sqrt{3}$, which is a known result for hot relativistic plasmas (Landau and Lifshitz, 1959).

Equation (58) shows that the normal modes correspond to an electroacoustic wave (given by $S=0$ ), Langmuir waves $(L=0)$, and sideband waves $\left(D_{ \pm}=0\right)$. In the presence of the pump wave $(\alpha \neq 0)$ these normal modes couple and give rise to the parametric decays. We will study this in detail in Sect. 4.4.

\subsection{Numerical analysis of the dispersion relation}

We now solve the dispersion relation Eq. (57) numerically. To this end, for given values of $\alpha$ and $\mu$, we choose a value of the normalized wave number of the pump wave, 


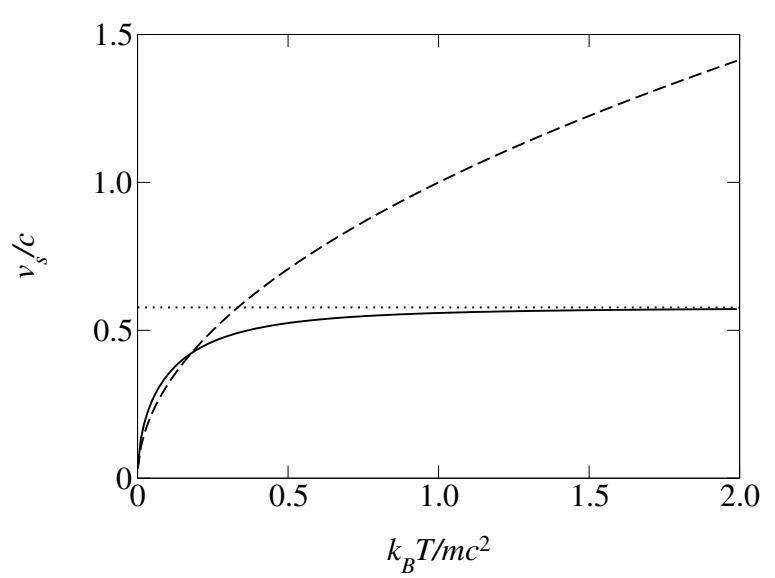

Fig. 11. Normalized sound velocity as a function of normalized temperature $\mu^{-1}=k_{B} T / m c^{2}$. Dashed line: classical result $(f=1)$, $v_{\mathrm{s}} / c=\sqrt{\mu^{-1}}$. Solid line: relativistic result (see Eq. 63). The horizontal dotted line represents the limit value $1 / \sqrt{3}$.

$y_{0}=k_{0} c / \Omega_{\mathrm{c}}$, and we solve the dispersion relation of the pump wave to get the normalized pump wave frequency $x_{0}=\omega_{0} / \Omega_{\mathrm{c}}$, as outlined in Sect. 2.1 and Asenjo et al. (2009). Notice that, in particular, this implies a choice of a branch for the pump wave, since a given $k_{0}$ may correspond to up to three values of $\omega_{0}$.

Then we solve Eq. (57). In Fig. 12 the pump wave ( $x_{0}$, $y_{0}$ ) has been chosen to lie in the Alfvén branch, near the origin. Figure 12a shows the solutions of Eq. (57) for $y_{0}=1$, $\omega_{\mathrm{p}} / \Omega_{\mathrm{c}}=1,1 / \mu=0.01$ and $\alpha=0$, which is equivalent to solving Eq. (58). There are 12 lines, two of which correspond to the electroacoustic modes that are the real solutions of $S=0$. They are labeled as $S_{+}$and $S_{-}$, corresponding to forward and backward propagating modes, respectively. Two other lines correspond to the Langmuir modes given by $L=0$, and labeled as $L_{+}$(forward) and $L_{-}$(backward). The other eight lines correspond to the eight real solutions of $D_{ \pm}=0$. Four of these lines are parabolic and correspond to the electromagnetic branches in Fig. 1, and they are labeled as $D_{ \pm}$in Fig. 12a. The two lines $p_{ \pm}$also correspond to solutions of $D_{ \pm}$, but they resonate at the sideband frequency $\omega_{ \pm}=\Omega_{\mathrm{c}} / f_{0}$, which in the cold case is the positron gyrofrequency (see Eqs. 61 and 62). In terms of the normalized longitudinal frequency $x=\omega / \Omega_{\mathrm{c}}$, the resonance occurs at $x= \pm\left(-x_{0}+1 / f_{0}\right)$, as shown in Fig. 12. Similarly, $e_{ \pm}$ are the two solutions that resonate at the sideband frequency $\omega_{ \pm}=-\Omega_{\mathrm{c}} / f_{0}$ (which, in the cold limit, is the electron gyrofrequency), that is, at $x= \pm\left(-x_{0}-1 / f_{0}\right)$.

We can see from Fig. 12a that there are several possible crossings between solutions of the dispersion relation. At these crossings, complex solutions can appear when $\alpha \neq 0$. Since the polynomial being solved has real coefficients, these solutions always occur as complex conjugate pairs, thus one of them has a positive imaginary frequency. Therefore, the
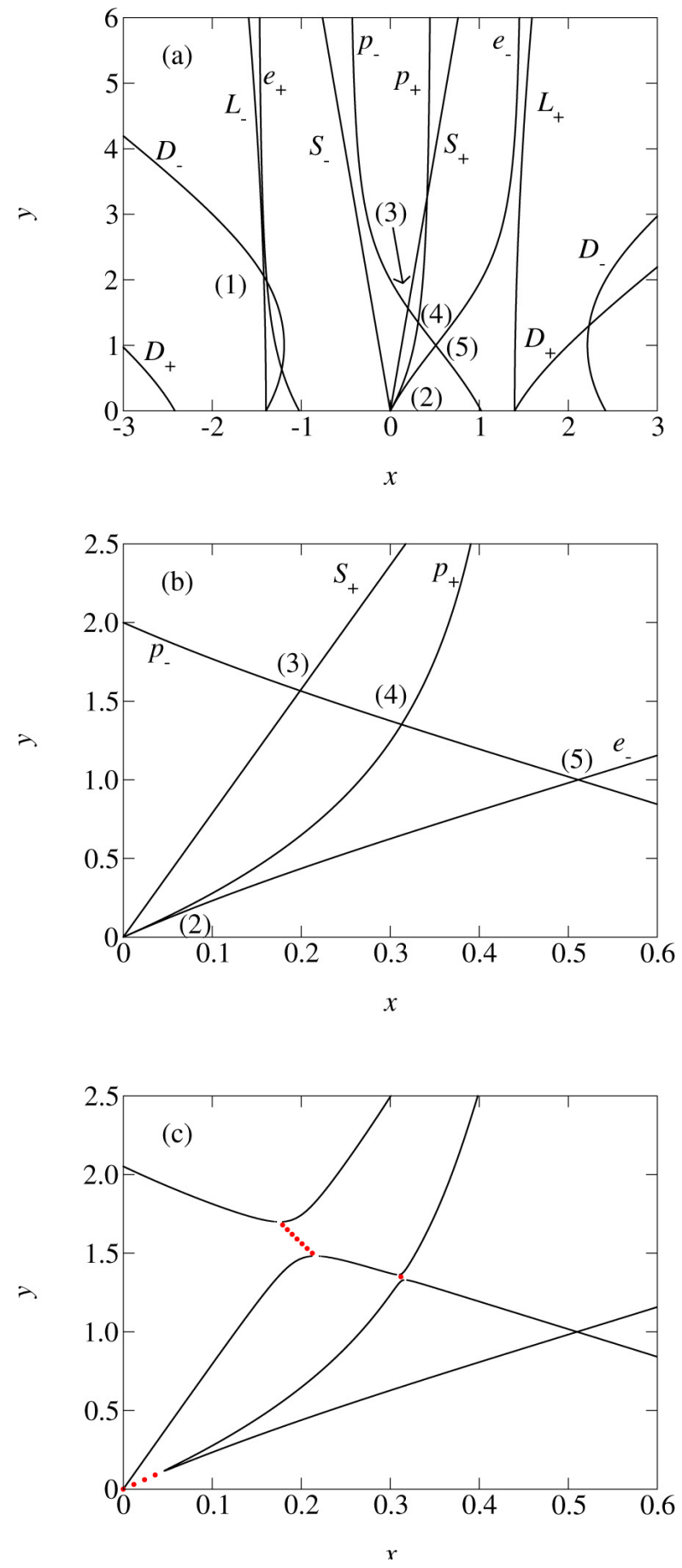

Fig. 12. (Color online) Solution of the dispersion relation Eq. (57). Normalized wave number $y=k c / \Omega_{\mathrm{c}}$ vs. normalized frequency $x=\omega / \Omega_{\mathrm{c}}$ for $y_{0}=1, \omega_{\mathrm{p}} / \Omega_{\mathrm{c}}=1,1 / \mu=0.01$. We take the pump wave at the Alfvén branch. (a) $\alpha=0$. (b) Sector of interest for $\alpha=0$, (c) $\alpha=0.1$. Dotted lines represent the real part of the solution when it is complex.

disappearance of real solutions when $\alpha \neq 0$ implies the presence of unstable waves, corresponding to the parametric decays of the pump wave. 
Only the crossings involving modes satisfying energy conservation $\omega_{1}+\omega_{2}=\ell \omega_{0}\left(\ell \in \mathbb{Z}^{+}\right)$can give rise to wave coupling when the pump wave is switched on $(\alpha \neq 0)$. They are marked with the labels from (1) to (5) in Fig. 12a. Crossing (1) is actually not considered here because it is unstable only in a narrow range of temperatures (around $\mu^{-1} \approx 0.01$ ) and its maximum growth rate is too small $\left(10^{-3}\right)$ compared with the other crossings. Thus we focus our attention on crossings (2)-(5) that are displayed in detail in Fig. 12b for $\alpha=0$. Crossing (3) corresponds to a resonant coupling $(\ell=1)$, and crossings (2), (4) and (5) correspond to nonresonant couplings.

Now we turn the pump wave on by considering $\alpha \neq 0$. In Fig. $12 \mathrm{c} \alpha=0.1$, and we notice that some crossings become gaps. This means that at these crossings we have complex solutions whose real parts are indicated as dotted lines, while the real solutions correspond to the continuous lines. Hence, we now have instabilities, indicating wave coupling. For example, crossing (3) gives rise to a wave coupling between $\left(S_{+}, p_{-}\right)$, corresponding to an ordinary decay instability in which the pump wave decays into a forward-propagating electroacoustic mode of frequency $\omega$ and a sideband wave of frequency $\omega_{-}$. Crossing (4) leads to a nonresonant wave coupling between $\left(p_{+}, p_{-}\right)$, in which the pump wave decays into two sideband waves of frequency $\omega_{-}$and $\omega_{+}$. In the case of crossing (5), there is no gap, and therefore no instability. Finally, crossing (2) represents an intersection of $\left(p_{+}, e_{-}\right)$at the origin, so it corresponds to an electromagnetic modulational instability (Longtin and Ö. Sonnerup, 1986).

As mentioned above, the appearance of gaps means that there exist modes whose frequency has a nonzero imaginary part. There are several possible decays simultaneously, but not all of them will be equally relevant. We can quantify this by calculating, for a given value of $\alpha$, the maximum imaginary part at each gap. By comparing these values for all gaps, we may then gain intuition regarding the relative importance of each decay for various values of $\alpha$. This is shown in Fig. 13. We define the imaginary part of the frequency as $\Gamma=\operatorname{Im}(x)$, and in each gap we seek its maximum value $\Gamma_{\max }$ (maximum growth rate), and we plot it against $\alpha$ and $1 / \mu$.

The first interesting feature we observe is that the resonant coupling (3) exhibits the expected linear dependence with $\alpha$, for small $\alpha$, while the nonresonant ones usually show a quadratic behavior for small values of $\alpha$. Let us also note that, for every pump wave amplitude, the maximum growth rate always occurs for $\mu^{-1} \leq 0.3$. In Fig. 13a, $\mathrm{b}$ and $\mathrm{d}$ we see that for $\mu^{-1}>0.1$ the instabilities disappear, but Fig. 13c shows that crossing (4) is still unstable, so for high temperatures crossing (4) is the dominant one.

Another interesting feature is that, for some crossings, there is a threshold value of $\alpha$ below which there is no instability, and that this threshold depends on the temperature. This can be seen in Fig. 14a, which shows that crossing (3) leads to an instability only if $\alpha>0.07$, approximately, when $1 / \mu=0.03$, whereas there is no threshold for the larger
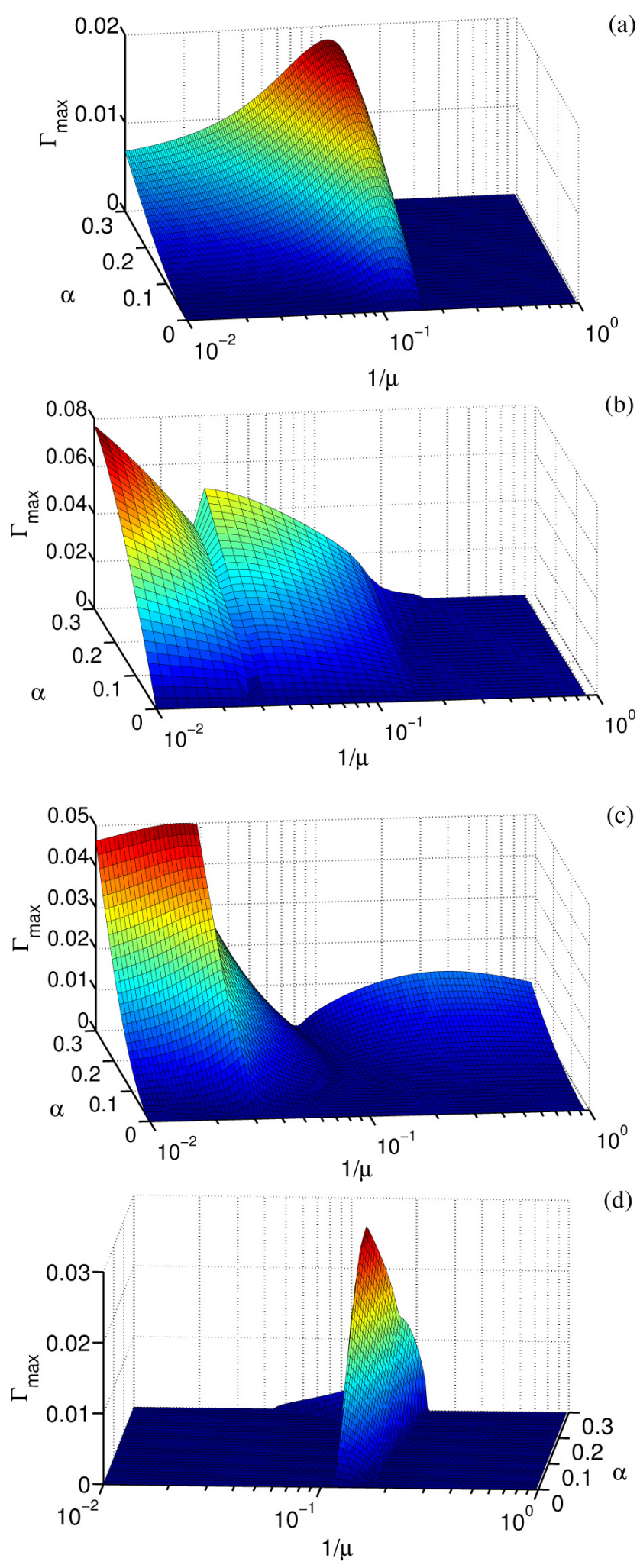

Fig. 13. (Color online) Maximum growth rate for various pump wave amplitudes $\alpha$ and temperatures $1 / \mu$. Notice that the scale is logarithmic scale for temperature. (a) Crossing (2), $\left(p_{+}, e_{-}\right)$. (b) Crossing (3), $\left(S_{+}, p_{-}\right)$. (c) Crossing $(4),\left(p_{+}, p_{-}\right)$. (d) Crossing (5), $\left(e_{-}, p_{-}\right)$. 

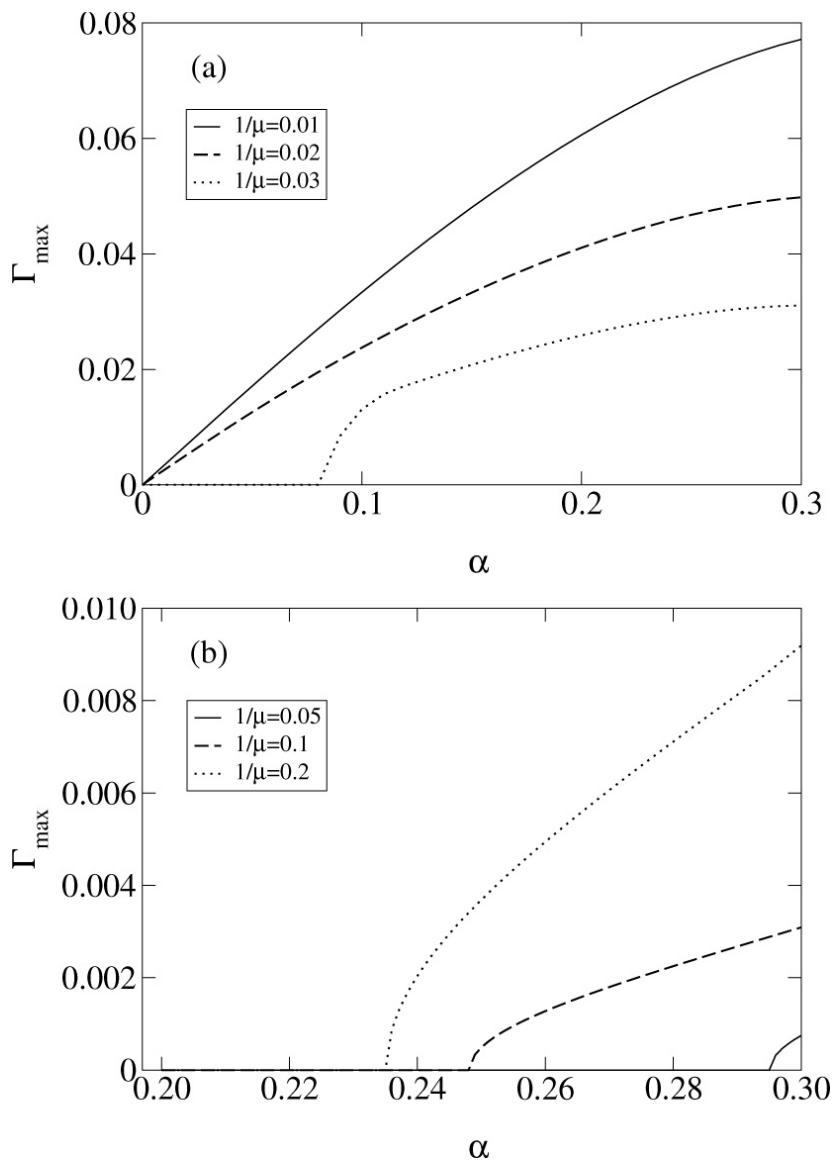

Fig. 14. Maximum growth rate vs. pump wave amplitude $\alpha$. Numerical labels for crossings are taken from Fig. 12. (a) Crossing (3), $\left(S_{+}, p_{-}\right)$. (b) Crossing (5), $\left(e_{-}, p_{-}\right)$.

temperatures shown in the plot. In the case of crossing (5) (Fig. 14b), there is a threshold for all temperatures. When temperature is increased, smaller values of $\alpha$ are needed to trigger the instability.

In Fig. 15 we have plotted the imaginary part of the frequency as a function of the wave number, for different values of $\alpha$ and a fixed value of $\mu$. This figure gives us an indication of the wave band over which the wave coupling occurs. We have labeled the instabilities as in Fig. 12a. In Fig. 15a we take $1 / \mu=0.01$, and as we expect for this range of values of $\alpha$, the resonant coupling (3) is the dominant one, being larger and broader than the other, nonresonant, couplings (2), (4) and (5). This also occurs in Fig. 15b, for $1 / \mu=0.02$. However, the wave band of active modes of crossing (3) has been significantly reduced, while the ones for crossings (2) and (4) have slightly increased. In Fig. 15, crossing (5) does not appear because it is stable for these temperatures.

Figure 15 suggests that the dominant coupling may be different for different values of $\alpha$ and $\mu$. We can appreciate this in Fig. 16, where we plot the maximum growth rate among all crossings for various temperatures and pump wave
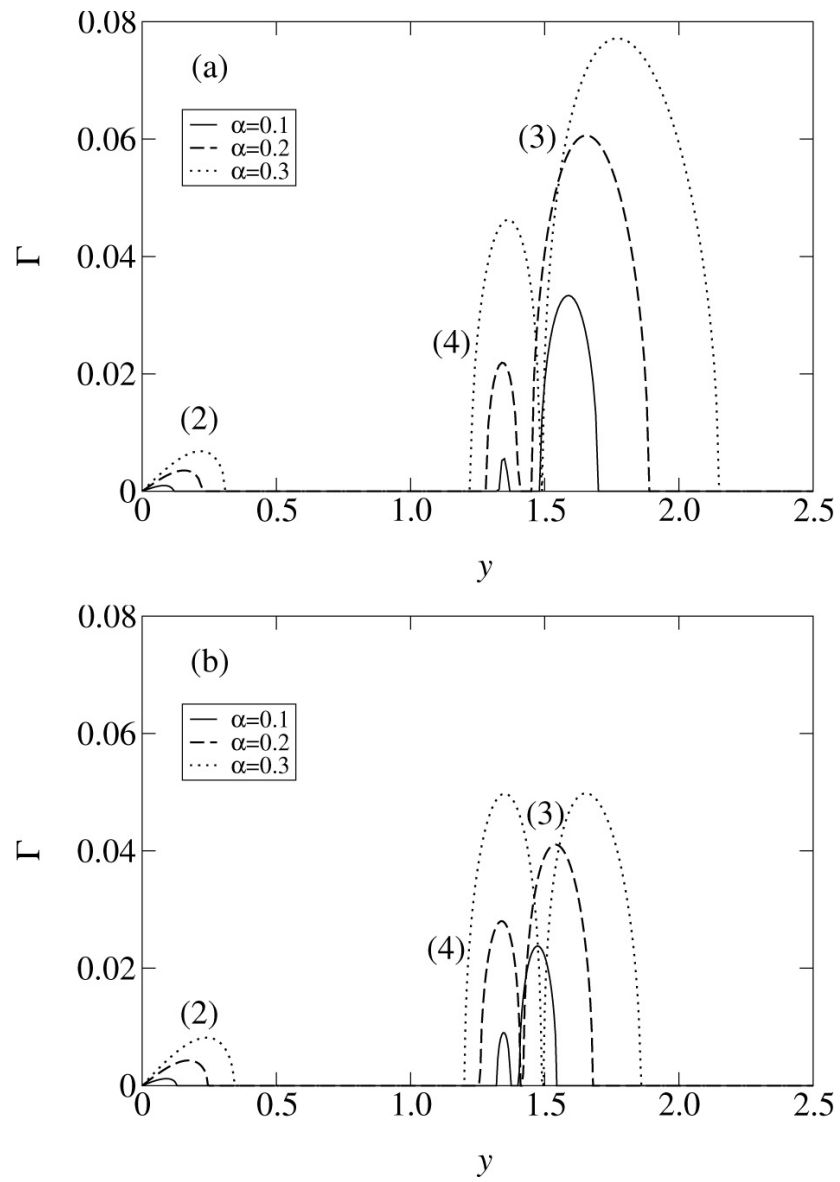

Fig. 15. Imaginary part of frequency vs. normalized wave number, for various values of the pump wave amplitude. Numerical labels for crossings are taken from Fig. 12. (a) $1 / \mu=0.01$. (b) $1 / \mu=$ 0.02 .

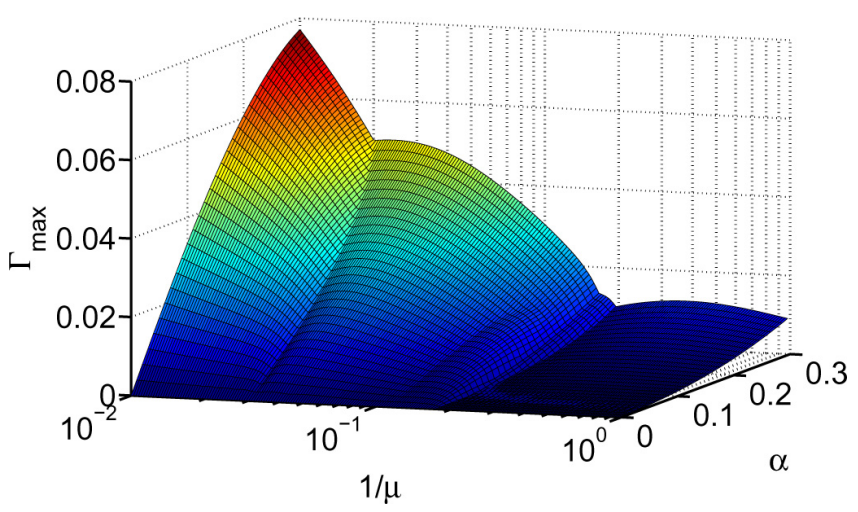

Fig. 16. (Color online) Maximum growth rate for various pump wave amplitudes $\alpha$ and temperatures $1 / \mu$, in logarithmic scale for temperature, considering all existent crossings.

amplitudes. Discontinuities in the derivative of the plotted surface are an indication of a change of regime, where the maximum growth rate changes from one crossing to another. 


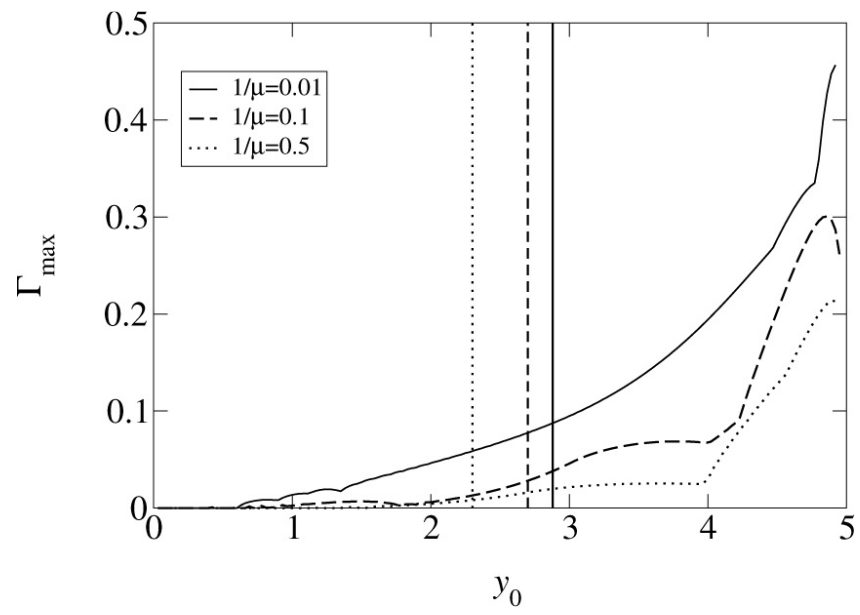

Fig. 17. Maximum growth rate vs. pump wave wave number, for $\alpha=0.04$ and various temperatures. The vertical lines indicate the value of $y_{0}$ at which $\mathrm{d} \omega / \mathrm{d} k=0$.

In particular, for low temperatures the dominant behavior is given by crossing (3), while for higher temperatures it is given by crossing (4), which turns out to be the only coupling present.

A similar analysis to the one presented in this section can be done for a pump wave in the electromagnetic branch and in the anomalous zone of the Alfvén branch (see López et al., 2012 for full details), with qualitatively similar results.

One interesting finding is that if the pump wave is in the Alfvén branch, $\Gamma_{\max }$ is about an order of magnitude larger than in Fig. 16, when the pump is a wave in the normal zone of the Alfvén branch. This difference can be observed in Fig. 17, where we set a pump wave amplitude $\alpha=0.04$ and a temperature $\mu^{-1}$, and the maximum growth rate is plotted for various values of $y_{0}$ in the Alfvén branch. When $y_{0}$ approaches the anomalous zone, the instability becomes larger.

\section{Numerical simulations}

In this section we present preliminary results from relativistic particle simulations that are consistent with the findings in Sects. 2, 3, and 4. Simulations have been done with a relativistic PIC code, with periodic boundary conditions, and initial particle thermal velocities have been taken from a Maxwell-Boltzmann-Jüttner distribution function. The detailed description of the code, as well as more complete results, will be presented elsewhere (López et al., 2014).

First, by choosing an initial condition where particles only have thermal velocities, we can obtain the normal mode dispersion relation from the power spectrum of magnetic fluctuations. This is shown in Fig. 18.

A good agreement with both fluid (Sect. 2) and kinetic (Sect. 3) results is found, as shown in Fig. 19, where the curves corresponding to the appropriate dispersion relations
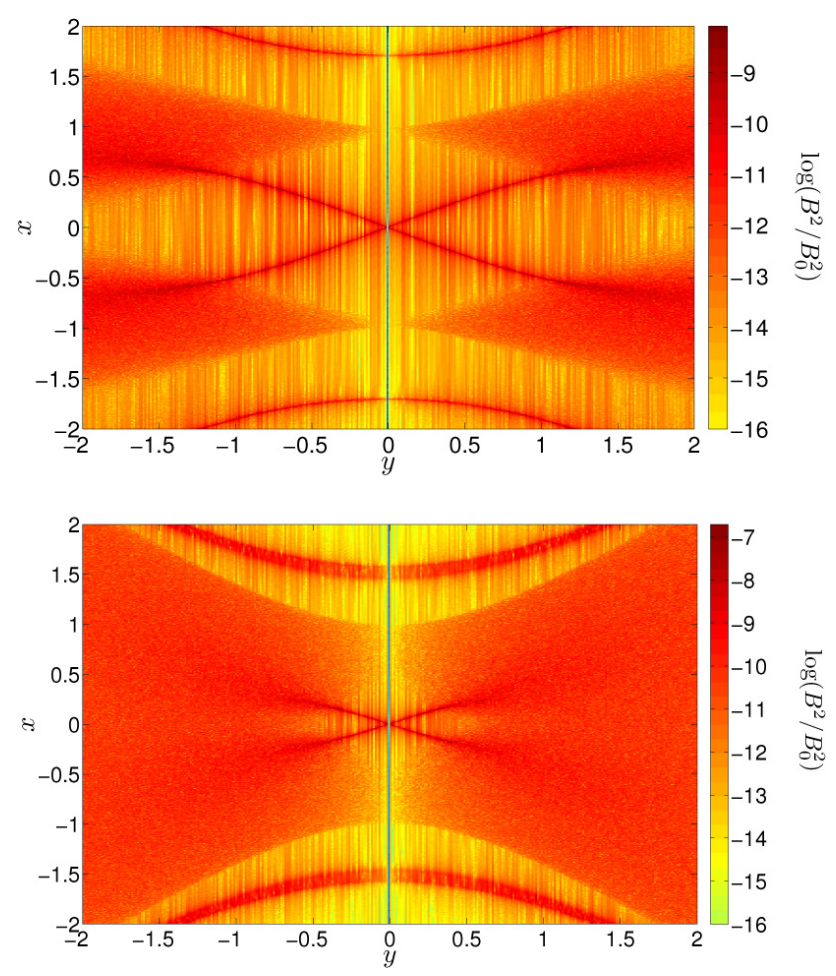

Fig. 18. Power spectrum of transverse magnetic fluctuations from a PIC simulation, for $\alpha=0.01, \omega_{\mathrm{p}} / \Omega_{\mathrm{c}}=1$. (a) $\mu=100$, (b) $\mu=7$.
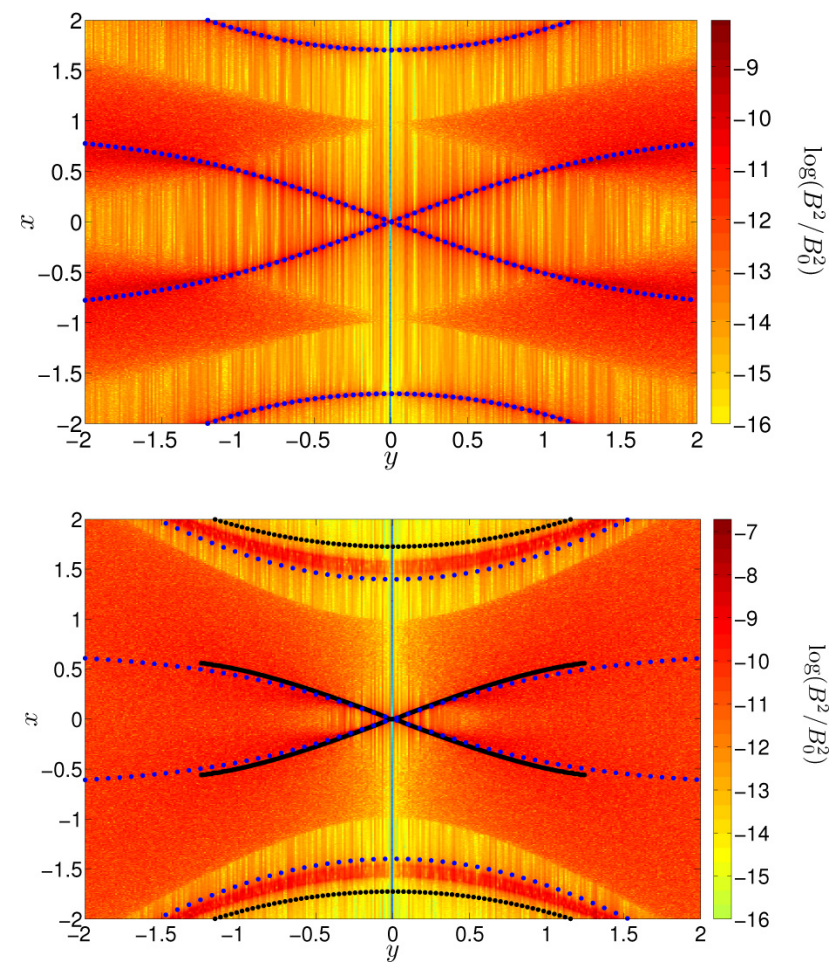

Fig. 19. Same as Fig. 18, but with curves from the analytical models superimposed. Blue dotted line: fluid dispersion relation, Eq. (21); black dotted line: kinetic dispersion relation, Eq. (40). (a) $\mu=100$, (b) $\mu=7$. 

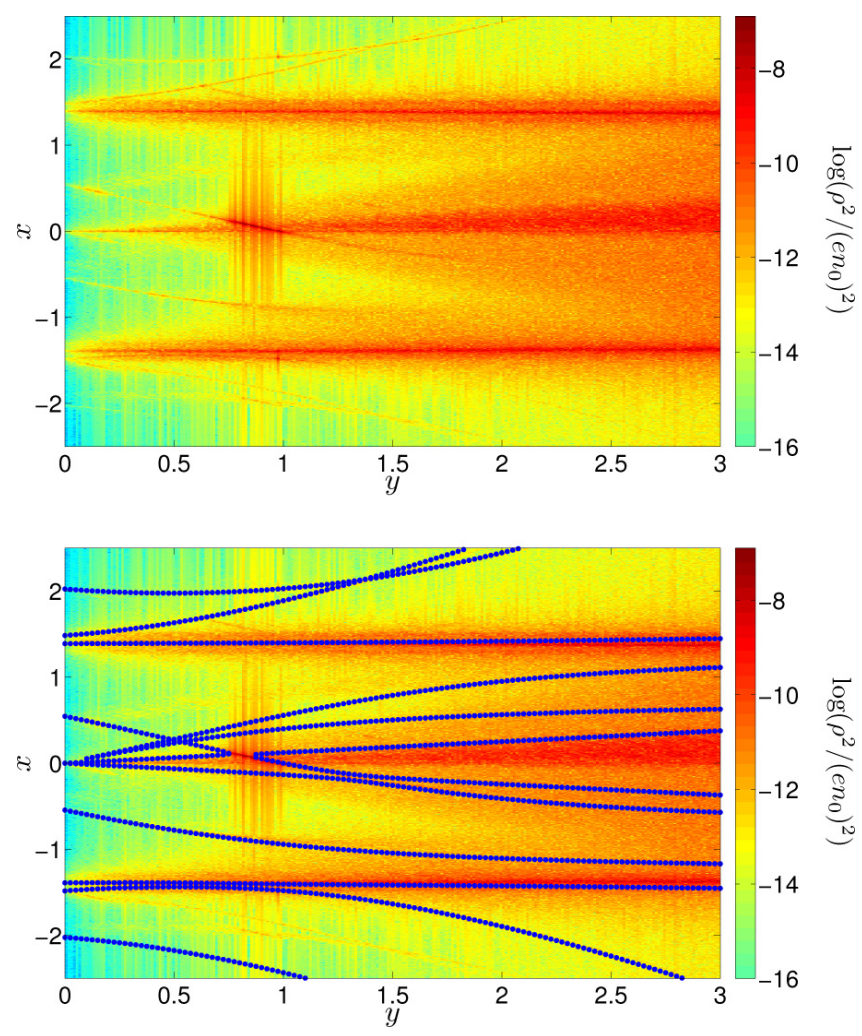

Fig. 20. Power spectrum of density fluctuations for the PIC simulation. The pump wave satisfies Eq. (21), with $\alpha=0.2$ and $\mu=100$, and is taken from the Alfvén branch, with $y_{0}=0.49, x_{0}=0.27$. (a) Simulation results; (b) lines correspond to the fluid dispersion relation of the parametric decays, Eq. (57), for the same parameters.

(Figs. 1 and 6) have been superimposed. In particular, both expected branches are observed, the electromagnetic and the Alfvén one. Regarding the Alfvén branch, in the simulation results it cannot be distinguished for wave numbers beyond a certain critical value, which suggests that it is damped due to kinetic effects.

Figure 19 also shows that the effective plasma frequency decreases with temperature (see Figs. 2 and 7), and that the Alfvén velocity decreases with temperature as well (see Eq. 23 and Fig. 8). When the effective plasma frequency and Alfvén velocity are measured from the simulation results, they turn out to be consistent with the fluid and kinetic models in Sects. 2 and 3.

We have also studied the parametric decays of a circularly polarized pump wave, following the model in Sect. 4. Figure 20a shows the power spectrum of density fluctuations, when the initial condition is a pump wave consistent with Eqs. (10) and (11), with $\alpha=0.2$ and $\mu=100$. Various modes corresponding to the parametric decays of the pump wave can be identified, with major wave activity near $y \sim 0.8$. In Fig. $20 \mathrm{~b}$ the dispersion relation for the parametric decays in the fluid model for the same parameters has been superim-
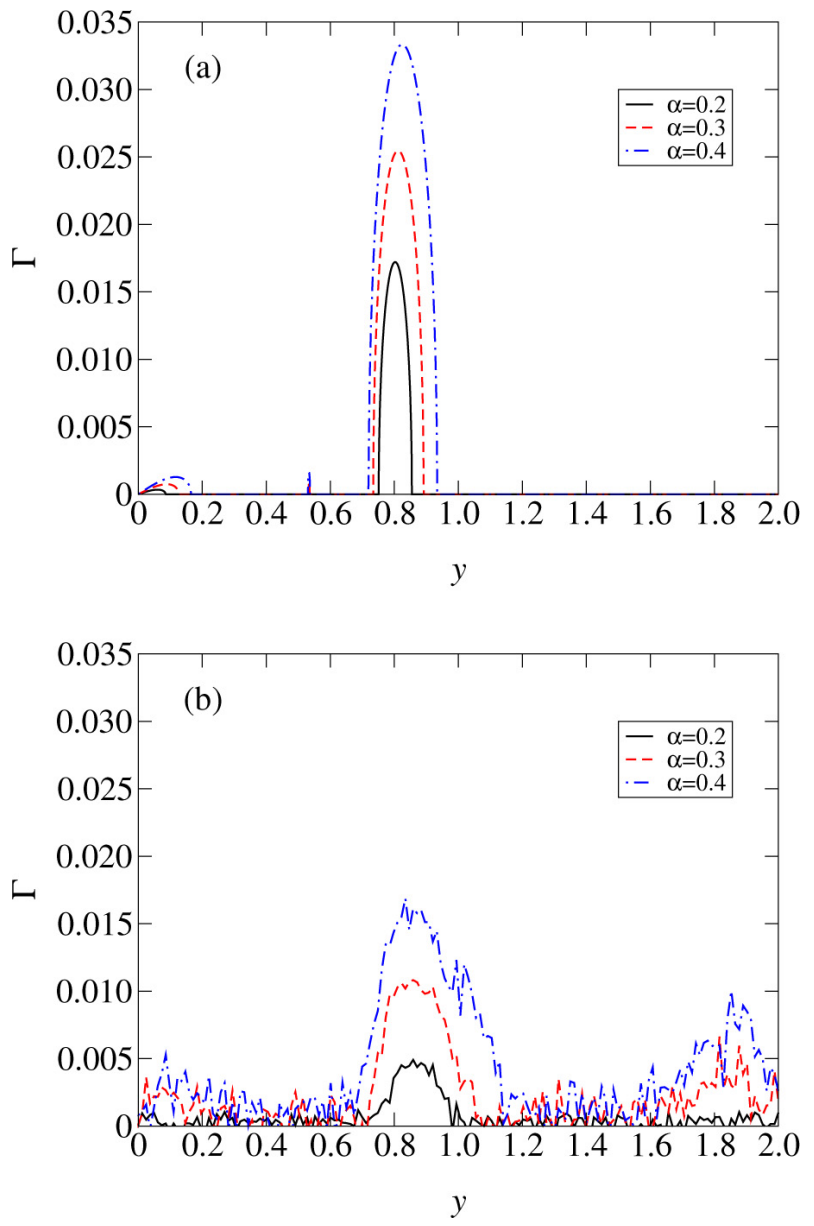

Fig. 21. Growth rate for the decay instability shown in Fig. 20. (a) Fluid result for the $\left(S_{+}, p_{-}\right)$crossing. (b) Simulation results for the same parameters.

posed, showing the consistency of the simulation and analytical results. In particular, the spectrum peak near $y \sim 0.8$ can be identified as the result of the nonlinear coupling between a sound wave $S_{+}$and a $p_{-}$sideband wave (see, e.g., crossing (3) in Fig. 12b).

Finally, growth rates for the decays can be estimated from the simulation. This is shown in Fig. 21, where the fluid and simulation results are shown. Again, both models are consistent.

\section{Conclusions}

We have studied the propagation of large-amplitude, circularly polarized electromagnetic waves in electron-positron plasmas, using fluid and kinetic models, and PIC simulations. Relativistic effects in the particle velocities have been introduced both for the quiver velocity induced by the wave, and for the random velocities induced by temperature. In the case of the fluid model, relativistic temperatures have been consistently introduced through a factor $f$ related to the enthalpy 
density. For the kinetic model and the simulations, relativistic temperatures have been introduced by assuming that the particles follow a Maxwell-Jüttner distribution function. Propagation of the wave has been assumed to occur along a constant background magnetic field.

In the case of the fluid model, an exact solution of the relativistic fluid equations can be found. A critical assumption is the fact that particle velocities are purely transverse with respect to the background magnetic field, which is true for circularly polarized electromagnetic waves of arbitrary amplitude. In particular, this means that no pressure or density fluctuations appear, and that $f$ is constant. We plan to explore the possibility of extending the above analysis to include a pressure tensor (Shadwick et al., 2004), a case in which we do not expect to find exact solutions, but which can certainly be studied numerically.

The fluid results show that two branches are present, an electromagnetic and an Alfvén branch. The electromagnetic branch has a lower cutoff at an effective plasma frequency $\omega_{\mathrm{p}}^{\mathrm{eff}}$, which decreases with temperature and wave amplitude due to the relativistic increase in effective mass of the particles.

The Alfvén branch, on the other hand, shows several interesting features. For very small wave numbers it is nondispersive, with an Alfvén velocity $v_{A}$ that depends only on temperature, not wave amplitude. As wave number increases, a maximum frequency $\omega_{\text {crit }}$, corresponding to a critical wave number $k_{\text {crit }}$, is reached. This upper cutoff depends both on wave amplitude and temperature. Thus, as temperature increases, Alfvén waves are confined to an ever narrower frequency bandwidth.

In a fluid model, important effects such as Landau damping are absent. Thus, a kinetic description is necessary. We have considered this problem too, studying the same problem, but based on the relativistic Vlasov equation, in order to account for kinetic effects in the direction of the magnetic field. The dispersion relation of the waves is found and numerically solved. Results are qualitatively consistent with the fluid model: the effective plasma frequency decreases, and the frequency range of the Alfvén branch is reduced when the temperature is increased, so that electromagnetic waves propagate in a larger frequency range with respect to the cold case, whereas for Alfvén waves the opposite occurs, being confined to a frequency range that is narrower than in the cold case.

However, quantitative results are different in both models. Regarding the electromagnetic branch, it is found that the effective plasma frequency is always larger in the kinetic model as compared with the fluid one. Thus, kinetic effects reduce the transparency of the plasma. As to the Alfvén branch, in both models it is found that the Alfvén speed decreases with temperature. In the fluid model, the Alfvén speed is zero for infinite temperature. However, in the kinetic case there is a critical, nonzero value of the temperature at which the Alfvén speed is zero. For temperatures above this critical value, the
Alfvén branch is suppressed. The critical value depends on the background magnetic field. For larger values of the magnetic field, the critical value increases, and thus Alfvén waves can propagate for larger temperatures.

We also studied the fluid dispersion relation of the waves for nonzero drift, thus generalizing the numerical results in Sect. 2. Besides, this analysis allowed us to study the dependence of the relativistic Lorentz factors on the drift, which was later used to study the kinetic model as detailed in Sect. 3.4.

We are also interested in the nonlinear evolution of the large-amplitude waves studied in Sects. 2 and 3. Thus, we have also studied the parametric decays of this wave, in the context of the fluid model. We study the appearance, the growth rate and instability range of the decays for various temperatures and pump wave amplitudes. We also consider pump waves in three possible regions: in the Alfvén branch (near the origin and in the anomalous zone), and in the electromagnetic branch. There are both resonant and nonresonant couplings when the pump wave is present. For high enough temperatures, though, most of the wave couplings disappear. For certain wave couplings there is a threshold pump wave amplitude, such that instability arises only above the threshold.

Finally, we have shown the consistency of the results above with those obtained from a relativistic PIC simulation. The existence of an electromagnetic and an Alfvén branch, the decrease in the effective plasma frequency and the Alfvén velocity with temperature, the confinement of the Alfvén branch to a narrow region of wave numbers and frequencies as temperature is increased, and the dispersion relation of the parametric decays, have been shown to agree qualitatively and in some cases quantitatively with both analytical models.

We expect to improve further on these results. For instance, we have not studied the damping due to kinetic effects. Another improvement would be to consider a kinetic model with a three-dimensional, rather than onedimensional, particle velocity distribution. Also, kinetic effects on the parametric decays need to be introduced, and some analytical results are available for the relativistic case (Muñoz et al., 2006). We have also studied the nonlinear evolution of electromagnetic waves in this system by means of the NLS equation, finding conditions for the existence of envelope solitons (Asenjo et al., 2012), localized structures that can also be found in our simulation runs, and we plan to investigate further these and other issues related to the propagation of nonlinear waves in relativistic pair plasmas elsewhere.

Acknowledgements. This work has been financially supported by FONDECyT under contract nos. 1121144 (V. Muñoz) and 1110135 (J. A. Valdivia). F. A. Asenjo is grateful to the Programa MECE Educación Superior for a doctoral scholarship, and Conicyt for a Becas Chile postdoctoral fellowship. M. Domínguez acknowledges financial support from Conicyt through a doctoral fellowship, 
contract no. 21100406. R. A. López thanks a doctoral fellowship from Conicyt, contract no. 21100839, and a Becas Chile fellowship, contract no. 75120086, for a doctoral internship at NASA/GSFC.

Edited by: A. C. L. Chian

Reviewed by: two anonymous referees

\section{References}

Asenjo, F. A., Muñoz, V., Valdivia, J. A., and Hada, T.: Circularly Polarized Wave Propagation in Magnetofluid Dynamics for Relativistic Electron-Positron Plasmas, Phys. Plasmas, 16, 122108, doi:10.1063/1.3272667, 2009.

Asenjo, F. A., Borotto, F., Chian, A. C.-L., Muñoz, V., Valdivia, J. A., and Rempel, E.: Self-Modulation of Nonlinear Waves in a Weakly Magnetized Relativistic ElectronPositron Plasma with Temperature, Phys. Rev. E, 85, 046406, doi:10.1103/PhysRevE.85.046406, 2012.

Bambah, B. A., Mahajan, S. M., and Mukku, C.: YangMills Magnetofluid Unification, Phys. Rev. Lett., 97, 072301, doi:10.1103/PhysRevLett.97.072301, 2006.

Barnes, A. and Hollweg, J. V.: Large-Amplitude Hydromagnetic Waves, J. Geophys. Res., 79, 2302-2318, 1974.

Berezhiani, V. I. and Mahajan, S. M.: Large Amplitude Localized Structures in a Relativistic Electron-Positron Ion Plasma, Phys. Rev. Lett., 73, 1110-1113, 1994.

Berezhiani, V. I. and Mahajan, S. M.: Large Relativistic Density Pulses in Electron-Positron-Ion Plasmas, Phys. Rev. E, 52, 19681979, 1995.

Beskin, V. S., Gurevich, A. V., and Istamin, Y. N.: Physics of the Pulsar Magnetosphere, Cambridge University Press, Cambridge, UK, 1993.

Björnsson, G., Abramowicz, M. A., Chen, X., and Lasota, J.-P.: Hot Accretion Disks Revisited, Astrophys. J., 467, 99-104, 1996.

Blaschke, D. B., Prozorkevich, A. V., Roberts, C. D., Schmidt, S. M., and Smolyansky, S. A.: Pair Production and Optical Lasers, Phys. Rev. Lett., 96, 140402, doi:10.1103/PhysRevLett.96.140402, 2006.

Chaudhary, R., Tsintsadze, N. L., and Shukla, P. K.: Nonlinear Propagation of Intense Electromagnetic Waves in a Hot ElectronPositron Plasma, J. Plasma Phys., 76, 875-886, 2010.

Chen, H., Wilks, S. C., Bonlie, J. D., Liang, E. P., Myatt, J., Price, D. F., Meyerhofer, D. D., and Beiersdorfer, P.: Relativistic Positron Creation Using Ultraintense Short Pulse Lasers, Phys. Rev. Lett., 102, 105001, doi:10.1103/PhysRevLett.102.105001, 2009.

Chen, H., Meyerhoferb, D. D., Wilks, S. C., Cauble, R., Dollar, F., Falk, K., Gregori, G., Hazi, A., Moses, E. I., Murphy, C. D., Myatt, J., Park, J., Seely, J., Shepherd, R., Spitkovsky, A., Stoeckl, C., Szabo, C. I., Tommasini, R., Zulick, C., and Beiersdorfer, P.: Towards Laboratory Produced Relativistic Electron-positron Pair Plasmas, High Energy Density Physics, 7, 225-229, 2011.

Curtis, M. F.: The Theory of Neutron Stars Magnetospheres, University of Chicago Press, Chicago, USA, 1991.

Domínguez, M., Muñoz, V., and Valdivia, J. A.: Thermal Effects on the Propagation of Large-Amplitude Electromagnetic Waves in Magnetized Relativistic Electron-Positron Plasma, Phys. Rev. E, 85, 056416, doi:10.1103/PhysRevE.85.056416, 2012.
Gibbons, G. W., Hawking, S., and Siklos, S. T. C. (Eds.): The Very Early Universe, Cambridge University Press, Cambridge, UK, 1985.

Gill, R. and Heyl, J. S.: Dispersion Relations for Bernstein Waves in a Relativistic Pair Plasma, Phys. Rev. E, 80, 036407, doi:10.1103/PhysRevE.80.036407, 2009.

Gomberoff, L. and Galvão, R. M. O.: Modulational Instability of a Circularly Polarized Wave in a Magnetized Electron-Positron Plasma With Relativistic Thermal Energies, Phys. Rev. E, 56, 4574, doi:10.1103/PhysRevE.56.4574, 1997.

Gomberoff, L., Gratton, F. T., and Gnavi, G.: Excitation and Parametric Decay of Electromagnetic Ion Cyclotron Waves in HighSpeed Solar Wind Streams, J. Geophys. Res., 99, 14717-14727, 1994.

Gomberoff, L., Muñoz, V., and Galvão, R. M. O.: Parametric Decays of a Linearly Polarized Electromagnetic Wave in an Electron-Positron Plasma, Phys. Rev. E, 56, 4581-4590, 1997.

Harding, A. K.: One-Photon Pair Annihilation in Magnetized Relativistic Plasmas, Astrophys. J., 300, 167-177, 1986.

Hardy, S. J. and Thoma, M. H.: Neutrino-Electron Processes in a Strongly Magnetized Thermal Plasma, Phys. Rev. D, 63, 025014, doi:10.1103/PhysRevD.63.025014, 2000.

Helander, P. and Ward, D. J.: Positron Creation and Annihilation in Tokamak Plasmas with Runaway Electrons, Phys. Rev. Lett., 90, 135004, doi:10.1103/PhysRevLett.90.135004, 2003.

Istomin, Y. N. and Sobyanin, D. N.: Electron-Positron Plasma Generation in a Magnetar Magnetosphere, Astron. Lett., 33, 660672, 2007.

Iwamoto, S. and Takahara, F.: Relativistic Outflow of ElectronPositron Pair Plasma from a Wien Equilibrium State, Astrophys. J., 565, 163-173, 2002.

Landau, L. D. and Lifshitz, E. M.: Fluid Mechanics,in: Course of Theoretical Physics, Vol. 6, Pergamon Press, Bristol, UK, 1959.

Lesch, H. and Pohl, M.: A Possible Explanation for Intraday Variability in Active Galactic Nuclei. Magnetic Reconnection and Coherent Plasma Emission, Astron. Astrophys., 254, 29-38, 1992.

Liang, E. P. T.: Electron-Positron Pair Production in Hot Unsaturated Compton Accretion Models around Black Holes, Astrophys. J., 234, 1105-1112, 1979.

Liu, Y. and Liu, S. Q.: Nonlinear Behavior of Electromagnetic Waves in Ultra-Relativistic Electron-Positron Plasmas, Contrib. Plasm. Phys., 58, 698-706, 2011.

Longtin, M. and Ö. Sonnerup, B. U.: Modulation Instability of Circularly Polarized Alfvén Waves, J. Geophys. Res., 91, 68166824, 1986.

López, R., Asenjo, F. A., Muñoz, V., and Valdivia, J. A.: Parametric Decays in Relativistic Magnetized Electron-Positron Plasmas with Relativistic Temperatures, Phys. Plasmas, 19, 082104, doi:10.1063/1.4742315, 2012.

López, R. A., Muñoz, V., Viñas, A. F., and Valdivia, J. A.: ParticleIn-Cell Simulation for Parametric Decays of a Circularly Polarized Alfvén Wave in Relativistic Electron-Positron Plasmas, in preparation, 2014.

Luo, Q., Melrose, D. B., and Fussell, D.: Wave Dispersion in Gyrotropic Relativistic Pulsar Plasmas, Phys. Rev. E, 66, 026405, doi:10.1103/PhysRevE.66.026405, 2002. 
Mahajan, S. M.: Temperature-Transformed "Minimal Coupling": Magnetofluid Unification, Phys. Rev. Lett., 90, 035001, doi:10.1103/PhysRevLett.90.035001, 2003.

Manchester, R. N. and Taylor, J. H.: Pulsars, W. H. Freeman, San Francisco, USA, 1977.

Matsukiyo, S. and Hada, T.: Parametric Instabilities of Circularly Polarized Alfvén Waves in Relativistic Electron-Positron Plasma, Phys. Rev. E, 67, 046406, doi:10.1103/PhysRevE.67.046406, 2003.

Muñoz, V.: Kinetic Effects on the Parametric Decays of Circularly Polarized Electromagnetic Waves in a Relativistic Pair Plasma, Phys. Plasmas, 11, 3497-3501, 2004.

Muñoz, V. and Gomberoff, L.: Parametric Decays of a Circularly Polarized Electromagnetic Wave in an Electron-Positron Plasma, Phys. Plasmas, 5, 3171-3179, 1998a.

Muñoz, V. and Gomberoff, L.: Parametric Decays of a Circularly Polarized Electromagnetic Wave in an Electron-Positron Magnetized Plasma, Phys. Rev. E, 57, 994-1004, 1998b.

Muñoz, V. and Gomberoff, L.: Electro-acoustic Damping Effects on the Parametric Decays of Electromagnetic Waves in ElectronPositron Plasmas, Phys. Plasmas, 7, 4916-4922, 2000.

Muñoz, V. and Gomberoff, L.: Kinetic Effects on the Parametric Decays of Circularly Polarized Electromagnetic Waves in an Electron-Positron Plasma, Phys. Plasmas, 9, 2534-2540, 2002.

Muñoz, V., Hada, T., and Matsukiyo, S.: Kinetic Effects on the Parametric Decays of Alfvén Waves in Relativistic Pair Plasmas, Earth Planets Space, 58, 1213-1217, 2006.

Pino, J., Li, H., and Mahajan, S.: Relaxed States in Relativistic Multifluid Plasmas, Phys. Plasmas, 17, 112112, doi:10.1063/1.3505326, 2010.

Piran, T.: Gamma-Ray Bursts and the Fireball Model, Phys. Rep., 314, 575-667, 1999.

Piran, T.: The Physics of Gamma-Ray Bursts, Rev. Mod. Phys., 76, 1143-1210, 2004.

Reynolds, C. S., Fabian, A. C., Celottid, A., and Rees, M. J.: The Matter Content of the Jet in M87: Evidence for an ElectronPositron Jet, Mon. Not. R. Astron. Soc., 283, 873-880, 1996.
Ruffini, R., Vereshchagin, G., and Xue, S.-S.: Electron-Positron Pairs in Physics and Astrophysics: From Heavy Nuclei to Black Holes, Phys. Rep., 487, 1-140, 2010.

Sawyer, R. F.: Hydrodynamics of an Electron-Positron Plasma near a Black Hole: Applications to Jet Formation, Phys. Rev. D, 77, 103011, doi:10.1103/PhysRevD.77.103011, 2008.

Shadwick, B. A., Tarkenton, G. M., and Esarey, E. H.: Hamiltonian Description of Low-Temperature Relativistic Plasmas, Phys. Rev. Lett., 93, 175002, doi:10.1103/PhysRevLett.93.175002, 2004.

Soto-Chavez, A. R., Mahajan, S. M., and Hazeltine, R. D.: Two-Fluid Temperature-Dependent Relativistic Waves in Magnetized Streaming Pair Plasmas, Phys. Rev. E, 81, 026403, doi:10.1103/PhysRevE.81.026403, 2010.

Sturrock, P. A.: A Model of Pulsars, Astrophys. J., 164, 529-556, 1971.

Tajima, T. and Taniuti, T.: Nonlinear Interaction of Photons and Phonons in Electron-Positron Plasmas, Phys. Rev. A, 42, 35873602, 1990.

Tatsuno, T., Berezhiani, V. I., Pekker, M., and Mahajan, S. M.: Angular Momenta Creation in Relativistic Electron-Positron Plasma, Phys. Rev. E, 68, 016409, doi:10.1103/PhysRevE.68.016409, 2003.

Usov, V. V.: Bare Quark Matter Surfaces of Strange Stars and $e^{+} e^{-}$ Emission, Phys. Rev. Lett., 80, 230-233, 1998.

Verdon, M. W. and Melrose, D. B.: Wave Dispersion in a Counterstreaming, Relativistic Thermal, Magnetized, Electron-Positron Plasma, Phys. Rev. E, 83, 056407, doi:10.1103/PhysRevE.83.056407, 2011.

Wardle, J. F. C., Homan, D. C., Ojha, R., and Roberts, D. H.: Electron-Positron Jets Associated with the Quasar 3C279, Nature, 395, 457-461, 1998.

White, T. R. and Lightman, A. P.: Hot accretion disks with electronpositron pairs, Astrophys. J., 340, 1024-1037, 1989.

Zank, G. P. and Greaves, R. G.: Linear and Nonlinear Modes in Nonrelativistic Electron-Positron Plasmas, Phys. Rev. E, 51, 6079-6090, 1995 